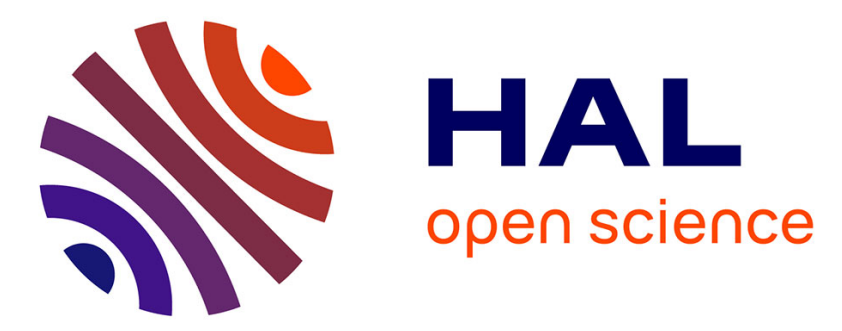

\title{
Ion transport through deformable porous media: derivation of the macroscopic equations using upscaling
}

Grégoire Allaire, Olivier Bernard, Jean-François Dufrêche, Andro Mikelic

\section{To cite this version:}

Grégoire Allaire, Olivier Bernard, Jean-François Dufrêche, Andro Mikelic. Ion transport through deformable porous media: derivation of the macroscopic equations using upscaling. 2015. hal-01215457

\author{
HAL Id: hal-01215457 \\ https://hal.science/hal-01215457
}

Preprint submitted on 14 Oct 2015

HAL is a multi-disciplinary open access archive for the deposit and dissemination of scientific research documents, whether they are published or not. The documents may come from teaching and research institutions in France or abroad, or from public or private research centers.
L'archive ouverte pluridisciplinaire HAL, est destinée au dépôt et à la diffusion de documents scientifiques de niveau recherche, publiés ou non, émanant des établissements d'enseignement et de recherche français ou étrangers, des laboratoires publics ou privés. 


\title{
Ion transport through deformable porous media: derivation of the macroscopic equations using upscaling*
}

\author{
Grégoire Allaire \\ E-mail: gregoire.allaire@polytechnique.fr \\ CMAP, Ecole Polytechnique, CNRS UMR 7641, Université Paris-Saclay, \\ F-91128 Palaiseau, France \\ Olivier Bernard \\ PHENIX, Université P. M. Curie - Paris 6 \\ CNRS UMR 8234 \\ Jean-François Dufrêche \\ E-mail: jean-francois.dufreche@univ-montp2.fr \\ Université de Montpellier 2, \\ Laboratoire Modélisation Mésoscopique et Chimie Théorique (LMCT) \\ Institut de Chimie Séparative de Marcoule ICSM UMR 5257 \\ CEA / CNRS / Université de Montpellier 2 / ENSCM Centre de Marcoule \\ Bât. 426 BP 1717130207 Bagnols sur Cèze Cedex, France \\ Andro Mikelić \\ E-mail: mikelic@univ-lyon1.fr \\ Université de Lyon, CNRS UMR 5208, \\ Université Lyon 1, Institut Camille Jordan, \\ 43, blvd. du 11 novembre 1918, 69622 Villeurbanne Cedex, France
}

October 9, 2015

\begin{abstract}
We study the upscaling or homogenization of the transport of a multicomponent electrolyte in a dilute Newtonian solvent through a deformable porous medium. The pore scale interaction between the flow and the structure deformation is taken into account. After a careful adimensionalization process, we first consider so-called equilibrium solutions, in the absence of external
\end{abstract}

\footnotetext{
${ }^{*}$ This research was partially supported by the project DYMHOM 2 (De la dynamique moléculaire, via l'homogénéisation, aux modèles macroscopiques de poroélasticité et électrocinétique) from the program NEEDS (Projet fédérateur Milieux Poreux MIPOR), GdR MOMAS and GdR PARIS. G. A. is a member of the DEFI project at INRIA Saclay Ile-de-France. The authors would like to thank Ch. Moyne (LEMTA, Nancy Université) and O. Bernard, V. Marry, B. Rotenberg and P. Turq from the Modélisation et Dynamique Multi-échelles team from the laboratory PHENIX (PHysicochimie des Electrolytes et Nanosystèmes InterfaciauX), UMR CNRS 8234, Université P. et M. Curie, for helpful discussions.
} 
forces, for which the velocity and diffusive fluxes vanish and the electrostatic potential is the solution of a Poisson-Boltzmann equation. When the motion is governed by a small static electric field and small hydrodynamic and elastic forces, we use O'Brien's argument to deduce a linearized model. Then we perform the homogenization of these linearized equations for a suitable choice of time scale. It turns out that the deformation of the porous medium is weakly coupled to the electrokinetics system in the sense that it does not influence electrokinetics although the latter one yields an osmotic pressure term in the mechanical equations. As a byproduct we find that the effective tensor satisfies Onsager properties, namely is symmetric positive definite.

pacs 02.30.Jr ; 47.61.Fg ; 47.56.+r ; 47.57.J- ; 47.70.Fw ; 47.90.+a ; 82.70.Dd ; 91.60.Pn . keywords Boltzmann-Poisson equation, elastic solid skeleton, homogenization, electro-osmosis.

\section{Introduction}

Effective modeling of the transport of an electrolyte through an electrically charged porous medium is an important and well-known multiscale problem in geosciences and porous materials modeling. It was studied by many authors and most of them assume that the $N$-component electrolyte, which is a dilute solution of $N$ species of ions in a fluid, saturates a rigid charged porous medium. Here, we depart from this usual assumption by considering a deformable porous medium.

Our motivation comes from the study of nuclear waste disposals. In such a case, the host material is clay, for which the pore size is typically a few hundred nanometers or even less. It means that the flow takes place in the electrostatic diffuse layer. To characterize and model the effective responses of porous materials to mechanical and physicochemical stresses, we first consider the pore modeling, including flow, convection, molecular diffusion, electrostatic effects, physical constraints on argillaceous rocks, and then apply the method of upscaling or homogenization. Our strategy is to exploit the natural occurrence of two very different spatial scales, namely the macroscopic scale and the scale of the average pore diameter and, by means of an asymptotic process, to deduce the effective behavior of a deformable porous solid, with small pores, when a charged liquid flows throughout, in the presence of an electric field. The main objective of the present research is to rigorously obtain homogenized models (i.e. valid at the macroscopic level) for the fluid-structure mixture in the presence of ion transport. The advantage of homogenization is clear: to obtain a mathematical model that does not rely on ad hoc assumptions, but based on physical reasoning. Note that the problems obtained for macroscopic variables may have little resemblance to the models developed from direct macroscopic approaches.

There is a long history of rigorous homogenization methods for the modeling of porous media. The macroscopic description of fluid flows in porous media frequently relies on the law of Darcy filtration. This model was justified using the theory of homogenization (see [5], [36], [38]). The fundamental assumption in these works is that the Darcy solid skeleton is rigid. The case of deformable porous media is more complicated and much research is devoted to this subject. Removing the assumption of rigid skeleton and replacing it with a linear elastic solid, a hierarchy of macroscopic models emerge [11] according to flow and deformation regimes. Historically the problem has first been studied by Biot, who proposed a linear macroscopic model, which couples the fluid pressure and the displacement of the solid skeleton (see the selection of Biot's papers in [39]). The model was justified under the assumption of linear elastic skeleton and infinitesimal deformations at pore scale. The last assumption greatly facilitates the use of the homogenization method (see [22] and previous works quoted therein) because the position and direction of the normal to the fluid-solid interface does not change. The assumption of small displacement of the fluid-solid interface, in relation to the pore size, also preserves other important properties such as the periodicity of the unit cell. 
If one is interested in transport through a quasi-static electrically charged porous medium, then there is also an extensive literature on the subject. We mention the series of articles by Adler et al. on the determination of the effective coefficients [2], [3], [10], [13], [15], [21], [35]. For rigid porous media, recent progresses have been achieved with the method of two-scale asymptotic expansions. Looker and Carnie gave in [20] an effective model with an Onsager tensor obtained as follows: they first apply O'Brien linearization [30] to the nonlinear Poisson-Nernst-Planck equations and, second, formally derive the homogenized model by means of two-scale asymptotic expansions applied to the linearized microscopic model. The rigorous derivation, with convergence of the homogenization process and a proof of the Onsager reciprocity principle, appeared in [6] (see also [7] for numerical simulations and a sensitivity analysis of the homogenized tensor). Note also the related works of Ray et al. [33] and Schmuck [37] with different asymptotic scalings.

After these recent advances in multi-scale modeling of electrokinetic effects in rigid porous media, it is natural to turn to deformable porous media, which represent a more realistic model (able to take into account, for example, swelling effects). Moyne and Murad have examined the case of nonlinear electro-osmosis periodic deformable porous media, without linearization, in a series of papers [23], [24], [25], [26], [27]. A formal two-scale asymptotic expansion has been applied and a coupled non-linear two-scale system of partial differential equations was obtained. Because of its complexity, this homogenized model is difficult to analyze or use in numerical practice. Moreover, the nonlinear character of Poisson-Nernst-Planck equations was preserved while the fluid-structure interaction was linearized. Note that, in the applied literature (see e.g. [28], [32]), most existing models are linear and correspond to larger pores. They are usually constructed assuming Onsager relations between the Darcy velocity and ionic current flows, on the one hand, and gradients of electrical potential, pressure and ionic concentrations, on the other hand. Then, the concentration gradients are added in Biot's equations, and the weighted potential gradient is added to the pressure gradient in the mass balance equation.

The content of our paper is the following. In Section 2 we introduce the microscopic model at the pore scale. It is a coupled system involving the Stokes equations for the incompressible fluid saturating the porous medium, the linearized elasticity equations for the deformable porous structure, Poisson equation for the electrostatic potential and the Nernst-Planck or convection-diffusion equations for the $N$ charged species in the fluid. The system is quasi-static which means that no inertial effects are taken into account except for the mass balance of each species. For simplicity, we consider an ideal model for the electrokinetic description of the electrolyte. One tricky aspect of the fluid-structure modeling is the choice of the advection velocity. We rely on the Arbitrary Lagrangian Eulerian formalism to settle this issue (more details are provided in Section 11 which reviews some classical notions in this setting). In Section 3 the equations of Section 2 are adimensionalized and the geometry of the porous medium is made precise. As usual in homogenization, the porous medium is assumed to be periodic and $\varepsilon$ denotes the small parameter which is the ratio of the periodic (microscopic) lengthscale and of a characteristic macroscopic lengthscale. We postpone to Section 6 the choice of the time scaling (which has to be related to the $\varepsilon$ parameter) since it is a delicate modeling issue which deserves its own section. In any case, such a choice is not yet necessary for establishing the so-called equilibrium solutions which are, by definition, stationary.

Section 4 is devoted to the computation of the equilibrium solutions, which correspond to vanishing applied forces. The only driving mechanism is the constant surface charge of the pore boundaries. In such a case, the system is reduced to the famous Poisson-Boltzmann equation which is non-linear monotone and classicaly admits a unique solution. This steady-state electrostatic distribution induces, through the Maxwell stress tensor (or osmotic Donnan pressure), a small elastic displacement of the solid skeleton of the porous medium. In Section 5, following the seminal work of O'Brien [30], 
we linearize the nonlinear model of Section 2 around the previously found equilibrium solutions. At this stage, we obtain a complicated, fully coupled, linear system of partial differential equations. It is now crucial to choose the time scaling. One of the main originality of our work is to perform a careful study of the possible time scalings in Section 6. Because of our view to possible applications in nuclear waste storage, we choose a characteristic time scale of the order of hundred thousands years which is clearly much larger than Terzaghi's time scale (related to vibrations of the porous medium and of the order of a few seconds) but turns out to be also larger than the diffusive time scale of the order of one month. As a consequence of these scaling assumptions, some time derivatives drop out in the linearized system (see Lemma 7) and it decouples into a standard electrokinetic system (as already studied in [20], [6], [7] plus an equation for the elastic perturbation and one for the potential perturbation.

It turns out that the decoupled electrokinetic system can be homogenized as in [20], [6], [7] and we recall these results in Section 7. In Theorem 10 we give the main convergence result while Proposition 11 gives the homogenized equations and states the Onsager reciprocity relations for the homogenized tensor. In Section 8 we give a new result on the homogenization of the perturbation of the electrostatic potential (see Theorem 14), which is necessary to deduce the homogenized equation for the elastic displacement. The latter one is obtained in Section 9 (see Theorem 17) and relies on the methods developed by Mikelić et al. [22], [17], [16], [12] to obtain the equations of Biot. The global homogenized system is assembled in Section 10, and its dimensional version is also proposed for practical applications. We also explain how to change the variables from ionic potentials to concentrations whic are more familiar to practionners. Eventually, Section 11 recalls some notations and results for the Arbitrary Lagrangian Eulerian formalism which is crucial to give a precise model of fluid-structure interaction at the pore scale.

\section{Equations of the model}

In this section we precisely describe the equations of our model, describing at the pore scale the electro-chemical interactions of an $N$-component electrolyte in a dilute Newtonian solvent within a deformable solid skeleton. The porous medium is denoted by $\Omega$, a bounded domain in $\mathbb{R}^{d}(d=2,3$ the space dimension): it is composed of the pore space $\Omega_{p}$, filled with the fluid, and of the solid skeleton $\Omega_{s}=\Omega \backslash \bar{\Omega}_{p}$. The fluid/solid interface is denoted by $\Gamma=\partial \Omega_{p} \cap \partial \Omega_{s}$. Our first modelling assumption is to consider a quasi-static regime as far as the mechanical equations are concerned. In other words, we neglect inertial effects for both the fluid and the solid (this is consistent with the time scale associated to a nuclear waste repository, see Section 6). Only the species mass balance will involve a time derivative term.

We start with the following Eulerian mass conservation law, for each species indexed by $i$,

$$
\frac{\partial n_{i}}{\partial t}+\operatorname{div}\left(\mathbf{j}_{i}+\mathbf{w} n_{i}\right)=0 \quad \text { in } \quad \Omega_{p}, \quad i=1, \ldots, N
$$

where $n_{i}$ is the $i$ th species concentration, $\mathbf{w}$ is the convective velocity and $\mathbf{j}_{i}$ is its migration-diffusion flux. (Calling (1) a mass balance equation is a slight abuse of language since it is rather a conservation law for the number of particles.) In a rigid porous medium the convective velocity is equal to the hydrodynamic velocity but, as we shall see, it is not the case in a deformable porous medium.

The migration-diffusion flux $\mathbf{j}_{i}$ is given by the following linear relationship

$$
\mathbf{j}_{i}=-\frac{n_{i} D_{i}^{0}}{k_{B} T}\left(\nabla \mu_{i}+z_{i} e \nabla \Psi\right), \quad i=1, \ldots, N,
$$

electroflux 
where $D_{i}^{0}$ is the diffusion coefficient for the $i$-th species, $z_{i}$ is the valence, $e$ is the elementary charge ( $-e$ is the electron charge), $k_{B}$ is the Boltzmann constant, $T$ is the absolute temperature and $\mu_{i}$ is the chemical potential given by

$$
\mu_{i}=\mu_{i}^{0}+k_{B} T \ln n_{i}
$$

where $\mu_{i}^{0}$ is a constant (the standard chemical potential expressed at infinite dilution). Furthermore, on the fluid/solid interface $\Gamma$ a no-flux condition holds true

$$
\mathbf{j}_{i} \cdot \nu=0 \quad \text { on } \Gamma, \quad i=1, \ldots, N .
$$

The electrostatic potential $\Psi$ is calculated from Poisson equation with the electric charge density as bulk source term

$$
\mathcal{E} \Delta \Psi=-e \sum_{j=1}^{N} z_{j} n_{j} \quad \text { in } \quad \Omega_{p}
$$

where $\mathcal{E}=\mathcal{E}_{0} \mathcal{E}_{r}$ is the dielectric constant of the solvent. The corresponding electrical field is $\mathbf{E}=-\nabla \Psi$. A surface charge $-\Sigma$ is assumed to be given at the pores boundaries $\Gamma$, namely the boundary condition reads

$$
\mathcal{E} \nabla \Psi \cdot \nu=-\Sigma \quad \text { on } \quad \Gamma
$$

where $\nu$ is the unit exterior normal to $\Omega_{p}$. The various parameters appearing in (1)-(6) are defined in Table 1. All quantities are given in SI units.

We assume that all valencies $z_{j}$ are different. If not, we lump together different ions with the same valency. Of course, for physical reasons, all valencies $z_{j}$ are integers. We rank them by increasing order and we assume that they are both anions and cations, namely positive and negative valencies,

$$
z_{1}<z_{2}<\ldots<z_{N}, \quad z_{1}<0<z_{N} .
$$

msa Remark 1. A more detailed, mathematically oriented, presentation of the fundamental concepts of electroosmotic flow in nanochannels can be found in the book [18] by Karniadakis et al., pages 447-470. The definitions (2) of the diffusive flux and (3) of the chemical potential correspond to the so-called ideal case. For simplicity we restrict ourselves to this simple setting, but most of the results remain valid for the more general model of the Mean Spherical Approximation (MSA). We refer to [14] for more details. A study of its upscaling in the case of a rigid porous medium can be found in [9]. More precisely, the MSA model replace (3) by

$$
\mu_{j}=\mu_{j}^{0}+k_{B} T \ln n_{j}+k_{B} T \ln \gamma_{j}\left(n_{1}, \ldots, n_{N}\right), \quad j=1, \ldots, N,
$$

with $\gamma_{j}$ being the activity coefficient of the species $j$. The diffusive flux (2) is replaced by

$$
\mathbf{j}_{i}=-\sum_{j=1}^{N} n_{i} L_{i j}\left(n_{1}, \ldots, n_{N}\right)\left(\nabla \mu_{j}+z_{j} e \nabla \Psi\right), \quad i=1, \ldots, N,
$$

where $L_{i j}\left(n_{1}, \ldots, n_{N}\right)$ is the (symmetric and positive definite) Onsager coefficient between $i$ and $j$. Contrary to the popular ideal model (2), which corresponds to the Poisson-Nernst-Planck equation, the MSA model is valid at high (molar) concentrations, because the non-ideality effect is taken into account both for the equilibrium (activity coefficients) and non-equilibrium (Onsager coefficients) coefficients [9]. For the sake of brevity we expose the ideal case in the sequel. 


\begin{tabular}{|l|l|l|}
\hline \hline & QUANTITY & CHARACTERISTIC VALUE \\
\hline $\mathrm{e}$ & electron charge & $1.6 \mathrm{e}-19 \mathrm{C}($ Coulomb $)$ \\
\hline$D_{i}^{0}$ & diffusivity of the $i$ th species & $D_{i}^{0} \in(1.333,2.032) \mathrm{e}-09 \mathrm{~m}^{2} / \mathrm{s}$ \\
\hline$k_{B}$ & Boltzmann constant & $1.38 \mathrm{e}-23 \mathrm{~J} / \mathrm{K}$ \\
\hline$n_{c}$ & characteristic concentration & $\left(6.0210^{24}, 6.0210^{26}\right) \mathrm{particles} / \mathrm{m}^{3}$ \\
\hline$T$ & temperature & $293^{\circ} \mathrm{K}(\mathrm{Kelvin})$ \\
\hline $\mathcal{E}$ & dielectric constant & $6.93 \mathrm{e}-10 \mathrm{C} /(\mathrm{mV})$ \\
\hline$\eta$ & dynamic viscosity & $1 \mathrm{e}-3 \mathrm{~kg} /(\mathrm{ms})$ \\
\hline$\ell$ & pore size & $5 \mathrm{e}-9 \mathrm{~m}$ \\
\hline$\lambda_{D}$ & Debye's length & $\sqrt{\mathcal{E} k_{B} T /\left(e^{2} n_{c}\right)} \in(0.042,0.42) \mathrm{nm}$ \\
\hline$z_{j}$ & $j$-th electrolyte valence & given integer \\
\hline$\Sigma$ & surface charge density & $0.129 \mathrm{C} / \mathrm{m}^{2}($ clays $)$ \\
\hline $\mathrm{f}$ & given applied force & $N / \mathrm{m}^{3}$ \\
\hline$\Psi_{c}$ & characteristic electrokinetic potential & $0.02527 \mathrm{~V}($ Volt $)$ \\
\hline$\Lambda$ & Young modulus & $7.3 \mathrm{e} 9 \mathrm{~Pa}$ \\
\hline
\end{tabular}

Table 1: Data description Data

The solute velocity is given by the incompressible Stokes equations with a forcing term made of an exterior hydrodynamical force $\mathbf{f}$ and of the electric force applied to the fluid thanks to the charged species

$$
\begin{gathered}
\nabla p-\eta \Delta \mathbf{v}=\rho_{f} \mathbf{f}-e \sum_{j=1}^{N} z_{j} n_{j} \nabla \Psi \quad \text { in } \quad \Omega_{p}, \\
\operatorname{div} \mathbf{v}=0 \quad \text { in } \quad \Omega_{p},
\end{gathered}
$$

where $\rho_{f}>0$ is the fluid density, $\mathbf{f}$ is the external body force, $\mathbf{v}$ is the fluid velocity and $p$ is the fluid pressure.

Adding to the usual viscous stress tensor the Maxwell tensor, we define the full stress tensor of the fluid phase

$$
\sigma^{f}=-p I+2 \eta e(\mathbf{v})+\mathcal{E}\left(\mathbf{E} \otimes \mathbf{E}-\frac{1}{2}|\mathbf{E}|^{2} I\right),
$$

where $\mathbf{E}=-\nabla \Psi$. With this notation the momentum equation (10) can be rewritten

$$
-\operatorname{div} \sigma^{f}=\rho_{f} \mathbf{f} \quad \text { in } \quad \Omega_{p},
$$

since the Poisson equation (5) yields

$$
\mathcal{E} \operatorname{div}\left(\mathbf{E} \otimes \mathbf{E}-\frac{1}{2}|\mathbf{E}|^{2} I\right)=-e \sum_{j=1}^{N} z_{j} n_{j} \nabla \Psi,
$$

electstress

which, in turn, implies (13).

We now turn to the linearized elasticity equations for the solid skeleton. We give here the result of a more detailed analysis in Section 11, which takes into account the difference of modelling, Eulerian for the fluid and Lagrangian for the solid, and fortunately simplifies under our standing assumption 
of small displacement and deformation. Let $\mathbf{u}^{s}$ be the elastic displacement. The strain tensor is $e\left(\mathbf{u}^{s}\right)=1 / 2\left(\nabla \mathbf{u}^{s}+\left(\nabla \mathbf{u}^{s}\right)^{*}\right)$, while the stress tensor is

$$
\sigma^{s}=A e\left(\mathbf{u}^{s}\right),
$$

where $A$ is a 4 th order symmetric positive definite tensor. As usual, we assume an isotropic tensor, $A_{i j k l}=2 \mu \delta_{i k} \delta_{j l}+\lambda \delta_{k l} \delta_{i j}$, where $\mu$ and $\lambda$ are the Lamé moduli. The linearized elasticity equations take the form

$$
-\operatorname{div} \sigma^{s}=\rho_{s} \mathbf{f} \quad \text { in } \quad \Omega_{s} .
$$

It remains to give the interface transmission conditions on $\Gamma$. As usual we enforce continuity of the velocities and of the normal stresses, namely

$$
\begin{array}{ccc}
\partial_{t} \mathbf{u}^{s}=\mathbf{v} & \text { on } & \Gamma, \\
\sigma^{f} \nu=\sigma^{s} \nu & \text { on } & \Gamma .
\end{array}
$$

The last delicate point is to define the convective velocity $\mathbf{w}$, appearing in the mass balance (1) for each species. As is common in fluid-structure modeling, we rely on an ALE (Arbitrary Lagrangian Eulerian) formalism, as explained in Section 11. In other words, extending the solid evelocity to the fluid part, the convective velocity is defined by

$$
\mathbf{w}=\mathbf{v}-\frac{\partial \mathbf{u}^{s}}{\partial t}
$$

All equations and interface conditions are now specified. The last step is to define boundary conditions on the exterior boundary $\partial \Omega$.

For simplicity we assume that $\Omega=(0, \mathcal{L})^{d}(d=2,3$ is the space dimension $), \mathcal{L}>0$ and at the outer boundary $\partial \Omega$ we set periodic boundary conditions

$$
\Psi+\Psi^{e x t}(x), n_{i}, \mathbf{u}^{s}, \mathbf{v} \text { and } p \text { are } \mathcal{L}-\text { periodic. }
$$

The applied exterior potential $\Psi^{e x t}(x)$ can typically be linear, equal to $\mathbf{E}^{\text {ext }} \cdot x$, where $\mathbf{E}^{\text {ext }}$ is an imposed electrical field. Note that the applied exterior force $\mathbf{f}$ appears both in the Stokes equations (10) and in the elasticity equations (16).

Due to the complexity of the geometry and of the equations, it is necessary for engineering applications to upscale the system (1)-(6), (10)-(11), (15)-(19). In order to do so, it is a common practice to assume that the porous medium has a periodic microstructure. More precisely, we assume that $\Omega, \Omega_{p}, \Omega_{s}$ are periodic of period $\ell>0$ in all space directions. One can interpret $\ell$ as the characteristic size of the pores in the porous medium. For simplicity we suppose $\mathcal{L} / \ell \in \mathbb{N}$, so that the domain is fully covered by entire cells. More detailed assumption will be made in the next section, after adimensionalization, for the simplicity of the exposition.

\section{Non-dimensional form}

We now give a non-dimensional form to the equations of Section 2 which will be later ready for linearization and homogenization. We follow the approach of our previous works [6], [7] (which were restricted to the case of a rigid porous medium) and we extend it to the case of a deformable medium following the adimensionalization process of the pore scale fluid-structure system as in [22]. 
We start by adimensionalizing the geometry. Let $\mathcal{L}_{c}$ be a characteristic size of the porous domain $\Omega$. We rescale the space variable by setting $\Omega^{\varepsilon}=\Omega_{p} / \mathcal{L}_{c}$ and $x^{\prime}=\frac{x}{\mathcal{L}_{c}}$ (we shall drop the primes for simplicity in the sequel). Introducing $L=\mathcal{L} / \mathcal{L}_{c}$, the rescaled domain is $(0, L)^{d}$, which we continue to denote by $\Omega$. We define a small adimensional parameter

$$
\varepsilon=\ell / \mathcal{L}_{c}<<1,
$$

which is precisely the period of the periodic porous domain $\Omega^{\varepsilon}$. We now define the geometrical structure of $\Omega$ and $\Omega^{\varepsilon}$.

Each period of $\Omega=(0, L)^{d}$ is homothetic to the unit cell $\left.Y=\right] 0,1\left[^{d}\right.$. Let $Y_{s}$ (the solid part) be a closed subset of $\bar{Y}$ and $Y_{F}=Y \backslash Y_{s}$ (the fluid part). We denote by $S$ the fluid/solid interface $S=\partial Y_{F} \cap \partial Y_{s}$. We make periodic repetition of $Y_{s}$ over $\mathbb{R}^{d}$ and, introducing $Y_{s}^{k}=Y_{s}+k, k \in \mathbb{Z}^{d}$, we define $E_{s}=\bigcup_{k \in \mathbb{Z}^{d}} Y_{s}^{k}$ which is a closed subset of $\mathbb{R}^{d}$. Similarly, $E_{F}=\mathbb{R}^{d} \backslash E_{s}$ in an open set in $\mathbb{R}^{d}$. We make the following assumptions on $Y_{F}$ and $E_{F}$ :

(H1) $Y_{F}$ is an open connected set of strictly positive measure, $Y_{s}$ has strictly positive measure too, and their interface $S$ is smooth,

(H2) $\quad E_{F}$ and the interior of $E_{s}$ are open sets with smooth boundaries. Moreover both $E_{F}$ and $E_{s}$ are connected.

The porous medium $\Omega=(0, L)^{d}$ is covered by a regular mesh of size $\varepsilon$, each cell being a cube $Y_{i}^{\varepsilon}$, with $1 \leq i \leq N(\varepsilon)=L^{d} \varepsilon^{-d}[1+o(1)]$. Each cube $Y_{i}^{\varepsilon}$ is homothetic to $Y$, by the linear homeomorphism $\Pi_{i}^{\varepsilon}$ (that is set by translation and homothety of ratio $1 / \varepsilon$ ). We define

$$
Y_{S_{i}}^{\varepsilon}=\left(\Pi_{i}^{\varepsilon}\right)^{-1}\left(Y_{s}\right) \quad \text { and } \quad Y_{f_{i}}^{\varepsilon}=\left(\Pi_{i}^{\varepsilon}\right)^{-1}\left(Y_{F}\right)
$$

For sufficiently small $\varepsilon>0$ we consider the set of indices $T_{\varepsilon}=\left\{k \in \mathbb{Z}^{d} \mid Y_{S_{k}}^{\varepsilon} \subseteq \Omega\right\}$ and define

$$
\Omega_{s}^{\varepsilon}=\bigcup_{k \in T_{\varepsilon}} Y_{S_{k}}^{\varepsilon}, \quad S_{\varepsilon}=\partial \Omega_{s}^{\varepsilon} \backslash \partial \Omega, \quad \Omega^{\varepsilon}=\Omega \backslash \Omega_{s}^{\varepsilon} .
$$

The domains $\Omega_{s}^{\varepsilon}$ and $\Omega^{\varepsilon}$ represent, respectively, the solid and fluid parts of the porous medium $\Omega$, while $S_{\varepsilon}$ is its fluid-solid interface.

We now turn to the adimensionalization of the physical variables. We denote by $n_{c}$ a characteristic concentration and we define the Debye length

$$
\lambda_{D}=\sqrt{\frac{\mathcal{E} k_{B} T}{e^{2} n_{c}}} .
$$

Following [18], we introduce the characteristic potential $\zeta$, the characteristic surface charge $\Sigma_{c}$, the characteristic electric field $E_{c}$ and the adimensional parameter $\beta$ (related to the Debye-Hückel parameter $\left.\kappa=1 / \lambda_{D}\right)$, as follows

$$
\zeta=\frac{k_{B} T}{e}, \quad \Sigma_{c}=\frac{\mathcal{E} \zeta}{\ell}, \quad E_{c}=\varepsilon \frac{\Sigma_{c}}{\mathcal{E}}, \quad \beta=\left(\frac{\ell}{\lambda_{D}}\right)^{2} .
$$

Note that the formula for $\Sigma_{c}$ directly comes from (6) and that the formula for $E_{c}$ is equivalent to $E_{c}=\zeta / \mathcal{L}_{c}$. We introduce a characteristic pressure and velocity as

$$
p_{c}=n_{c} k_{B} T \quad \text { and } \quad v_{c}=\varepsilon \frac{\ell k_{B} T n_{c}}{\eta}=\frac{\varepsilon^{2} p_{c} \mathcal{L}_{c}}{\eta} .
$$


Let $\Lambda$ be the characteristic size of the elastic moduli and $u_{c}^{s}$ the characteristic elastic displacement. In the porous medium we expect the pressure to be the dominant part of the fluid stress and to balance the elastic contact force at the interfaces. Consequently, the interface condition (18) implies that we choose $\Lambda$ and $u_{c}^{s}$ related by

$$
p_{c}=\frac{\Lambda u_{c}^{s}}{\mathcal{L}_{c}} .
$$

swell1 Remark 2. Introducing an adimensionalized displacement $\mathbf{u}^{\varepsilon}=\mathbf{u}^{s} / u_{c}^{s}$, the deformation gradient (rigorously defined in Section 11) scales as $\mathbf{F}_{\varepsilon}=I+\frac{u_{c}^{s}}{\mathcal{L}_{c}} \nabla_{x^{\prime}} \mathbf{u}^{\varepsilon}$ and its Jacobian determinant as $J^{\varepsilon}=\operatorname{det} \mathbf{F}_{\varepsilon}$. Thus we deduce

$$
\mathbf{F}_{\varepsilon}=I+\frac{n_{c} k_{B} T}{\Lambda} \nabla_{x^{\prime}} \mathbf{u}^{\varepsilon} .
$$

Using the data from Table 1 yields $\frac{n_{c} k_{B} T}{\Lambda} \approx 3.5 e-5$. Therefore, it justifies our approximation $\mathbf{F}_{\varepsilon} \approx I$ and $J^{\varepsilon} \approx 1$, as explained in Section 11. In other words we can use a simple linear model in an ALE (Arbitrary Lagrangian Eulerian) formulation.

The imposed forcing terms are the surface charge density $\Sigma$ (having the characteristic value $\Sigma_{c}$ ), the static electrical potential $\Psi^{e x t}$ and the applied fluid force $\mathbf{f}$. We then introduce adimensionalized forcing terms

$$
\Psi^{e x t, *}=\frac{\Psi^{e x t}}{\zeta}, \quad \mathbf{f}^{*}=\frac{\mathbf{f} \mathcal{L}_{c}}{p_{c}}, \quad \Sigma^{*}=\frac{\Sigma}{\Sigma_{c}}, \quad N_{\sigma}=\frac{e \Sigma_{c} \ell}{\mathcal{E} k_{B} T}=O(1),
$$

and adimensionalized unknowns

$$
p^{\varepsilon}=\frac{p}{p_{c}}, \mathbf{v}^{\varepsilon}=\frac{\mathbf{v}}{v_{c}}, \mathbf{w}^{\varepsilon}=\frac{\mathbf{w}}{v_{c}}, \mathbf{u}^{\varepsilon}=\frac{\mathbf{u}^{s}}{u_{c}^{s}}, \Psi^{\varepsilon}=\frac{\Psi}{\zeta}, n_{j}^{\varepsilon}=\frac{n_{j}}{n_{c}}, \mathbf{j}_{j}^{\varepsilon}=\frac{\mathbf{j}_{j} \mathcal{L}_{c}}{n_{c} D_{j}^{0}},
$$

where $D_{j}^{0}$ is the characteristic diffusion coefficient of the $j$-th species. For the dimensionless electrical field we set $\mathbf{E}^{\varepsilon}=\nabla \Psi^{\varepsilon}$, which is consistent with our definition of the characteristic electric field $E_{c}$.

Concerning time we introduce three time scales. First, $\mathcal{T}_{c}$ is the characteristic time scale of observation. It is used to rescale the time variable by setting $t^{\prime}=\frac{t}{\mathcal{T}_{c}}$ (we shall drop the primes for simplicity in the sequel). Second, we introduce a diffusion time scale

$$
\mathcal{T}_{d}=\frac{\mathcal{L}_{c}^{2}}{D^{0}}
$$

pressuresize

Third, we define the so-called Terzaghi's time scale

$$
\mathcal{T}_{T}=\frac{\eta}{\varepsilon^{2} \Lambda},
$$

which is related to the equilibrium between fluid and solid stresses at the interface.

We decompose the fluid velocity $\mathbf{v}$ as

$$
\mathbf{v}=v_{c}\left(\mathbf{w}^{\varepsilon}+\frac{\eta}{\varepsilon^{2} \mathcal{T}_{c} \Lambda} \frac{\partial \mathbf{u}^{\varepsilon}}{\partial t^{\prime}}\right)=v_{c}\left(\mathbf{w}^{\varepsilon}+\frac{\mathcal{T}_{T}}{\mathcal{T}_{c}} \frac{\partial \mathbf{u}^{\varepsilon}}{\partial t^{\prime}}\right),
$$

where $\mathbf{w}^{\varepsilon}$ is the relative flow velocity and $\frac{\partial \mathbf{u}^{\varepsilon}}{\partial t^{\prime}}$ is the extended structure velocity. Note that the above definitions implies that

$$
\frac{\mathcal{E} \zeta^{2}}{\mathcal{L}_{c}^{2}}=\frac{\varepsilon^{2}}{\beta} p_{c},
$$


which is equivalent to say that the Maxwell stress tensor is of the same order of magnitude than the viscous stress tensor (this relation is useful in deriving (25) below).

The dimensionless equations for hydrodynamical and electrostatic part are thus

$$
\begin{gathered}
\sigma^{f, \varepsilon}=-p^{\varepsilon} I+2 \varepsilon^{2} e\left(\mathbf{w}^{\varepsilon}\right)+2 \varepsilon^{2} \frac{\mathcal{T}_{T}}{\mathcal{T}_{c}} e\left(\frac{\partial \mathbf{u}^{\varepsilon}}{\partial t}\right)+\frac{\varepsilon^{2}}{\beta}\left(\mathbf{E}^{\varepsilon} \otimes \mathbf{E}^{\varepsilon}-\frac{1}{2}\left|\mathbf{E}^{\varepsilon}\right|^{2} I\right) \quad \text { in } \Omega^{\varepsilon}, \\
-\operatorname{div} \sigma^{f, \varepsilon}=\mathbf{f}^{*} \text { in } \Omega^{\varepsilon}, \\
\mathbf{w}^{\varepsilon}=0 \text { on } \partial \Omega^{\varepsilon} \backslash \partial \Omega, \quad \operatorname{div}\left(\mathbf{w}^{\varepsilon}+\frac{\mathcal{T}_{T}}{\mathcal{T}_{c}} \frac{\partial \mathbf{u}^{\varepsilon}}{\partial t}\right)=0 \quad \text { in } \Omega^{\varepsilon}, \\
-\operatorname{div} \sigma^{s, \varepsilon}=\mathbf{f}^{*} \text { in } \Omega_{s}^{\varepsilon}=\Omega^{\varepsilon} \backslash \bar{\Omega}^{\varepsilon}, \\
\sigma^{s, \varepsilon}=A e\left(\mathbf{u}^{\varepsilon}\right) \quad \text { in } \Omega_{s}^{\varepsilon}, \\
\sigma^{f, \varepsilon} \nu=\sigma^{s, \varepsilon} \nu \text { on } \partial \Omega^{\varepsilon} \backslash \partial \Omega, \\
-\varepsilon^{2} \Delta \Psi^{\varepsilon}=\beta \sum_{j=1}^{N} z_{j} n_{j}^{\varepsilon}(x) \quad \text { in } \Omega^{\varepsilon} ; \quad \varepsilon \nabla \Psi^{\varepsilon} \cdot \nu=-\Sigma^{*} N_{\sigma} \text { on } \partial \Omega^{\varepsilon} \backslash \partial \Omega, \\
\left(\Psi^{\varepsilon}+\Psi^{e x t, *}\right), \quad \mathbf{u}^{\varepsilon}, \quad \mathbf{w}^{\varepsilon} \text { and } p^{\varepsilon} \text { are } L-\text { periodic in } x .
\end{gathered}
$$

Concerning the transport equation, we defined the Peclet number for the $j$-th species by

$$
\mathrm{Pe}_{j}=\frac{v_{c} \mathcal{L}_{c}}{D_{j}^{0}}=\frac{\ell^{2} k_{B} T n_{c}}{\eta D_{j}^{0}},
$$

which shall be assumed to be of order 1 as $\varepsilon$ goes to zero. Using the diffusion time (24), we obtain the dimensionless form of equation (1):

$$
\begin{gathered}
\frac{\mathcal{T}_{d}}{\mathcal{T}_{c}} \frac{\partial n_{i}^{\varepsilon}}{\partial t}+\mathrm{Pe}_{i} \operatorname{div}\left(\mathbf{w}^{\varepsilon} n_{i}^{\varepsilon}\right)+\operatorname{div} \mathbf{j}_{i}^{\varepsilon}=0 \quad \text { in } \quad \Omega^{\varepsilon}, i=1, \ldots, N, \\
\mathbf{j}_{i}^{\varepsilon} \cdot \nu=0 \text { on } \partial \Omega^{\varepsilon} \backslash \partial \Omega, \quad \text { with } \quad \mathbf{j}_{i}^{\varepsilon}=-n_{i}^{\varepsilon} \nabla \ln \left(n_{i}^{\varepsilon} e^{z_{i} \Psi^{\varepsilon}}\right), \quad i=1, \ldots, N .
\end{gathered}
$$

Remark 3. So far we introduced three time scales: the characteristic time scale $\mathcal{T}_{c}$, the diffusion time scale $\mathcal{T}_{d}=\mathcal{L}_{c}^{2} / D^{0}$ and the Terzaghi's time scale $\mathcal{T}_{T}=\eta /\left(\varepsilon^{2} \Lambda\right)$. Since we are interested in the flow through a deformable porous medium and not in studying vibrations, we assume that $\mathcal{T}_{c}>>$ $\mathcal{T}_{T}$. Furthermore we note that $\mathcal{T}_{T} / \mathcal{T}_{d}=\left(\eta D^{0}\right) /\left(\Lambda \ell^{2}\right) \approx 1 e-5$ according to the values of Table 1. Therefore, we also have $\mathcal{T}_{d}>>\mathcal{T}_{T}$.

There is another possible time scale $\mathcal{T}_{v}=\mathcal{L}_{c} / v_{c}$ associated to convective effects. In the sequel we shall choose $\mathcal{T}_{c}=\mathcal{T}_{v}$.

\section{Equilibrium solution}

The goal of this section is to find a so-called equilibrium solution of system (25)-(34) when the exterior forces are vanishing $\mathbf{f}=0$ and $\Psi^{e x t}=0$. However, the surface charge density $\Sigma^{*}$ is not assumed to vanish or to be small. This equilibrium solution will be a reference solution around which we shall linearize system (25)-(34) in the next section. Then we will perform the homogenization of the (partially) linearized system in Section 7 . We denote by $n_{i}^{0, \varepsilon}, \Psi^{0, \varepsilon}, \mathbf{u}^{0, \varepsilon}, \mathbf{w}^{0, \varepsilon}, p^{0, \varepsilon}$ the equilibrium quantities. 
In the case $\mathbf{f}=0$ and $\Psi^{\text {ext }}=0$, one can find an equilibrium solution by forcing all unknowns to be time-independent and choosing a zero fluid velocity and all diffusive fluxes equal to zero. More precisely, we require

$$
\mathbf{w}^{0, \varepsilon}=0, \quad \mathbf{u}^{0, \varepsilon}=\mathbf{u}^{0, \varepsilon}(x) \quad \text { and } \quad \nabla \ln \left(n_{j}^{0, \varepsilon} e^{z_{j} \Psi^{0, \varepsilon}}\right)=0,
$$

which obviously implies that $\mathbf{j}_{i}^{0, \varepsilon}=0$ and (33)-(34) are satisfied. From $\nabla \ln \left(n_{j}^{0, \varepsilon} e^{z_{j} \Psi^{0, \varepsilon}}\right)=0$ we deduce that there exist constants $n_{j}^{0}(\infty)>0$ such that

$$
n_{j}^{0, \varepsilon}(x)=n_{j}^{0}(\infty) \exp \left\{-z_{j} \Psi^{0, \varepsilon}(x)\right\} .
$$

The value $n_{j}^{0}(\infty)$ is the infinite dilute concentration which will be later assumed to satisfy the bulk electroneutrality condition for zero potential.

Then the electrostatic equation (31) reduces to the Poisson-Boltzmann equation which is a nonlinear partial differential equation for the sole unknown $\Psi^{0, \varepsilon}$

$$
\left\{\begin{array}{l}
-\varepsilon^{2} \Delta \Psi^{0, \varepsilon}=\beta \sum_{j=1}^{N} z_{j} n_{j}^{0}(\infty) \exp \left\{-z_{j} \Psi^{0, \varepsilon}\right\} \text { in } \Omega^{\varepsilon} \\
\varepsilon \nabla \Psi^{0, \varepsilon} \cdot \nu=-N_{\sigma} \Sigma^{*} \text { on } \partial \Omega^{\varepsilon} \backslash \partial \Omega, \Psi^{0, \varepsilon} \text { is } L-\text { periodic. }
\end{array}\right.
$$

From a physical point of view, it is desired that the solution of (37) vanishes, i.e., $\Psi^{0, \varepsilon}=0$, when the surface charges are null, i.e., $\Sigma^{*}=0$. Therefore, following the literature, we impose the bulk electroneutrality condition

$$
\sum_{j=1}^{N} z_{j} n_{j}^{0}(\infty)=0 .
$$

The existence and uniqueness of a solution to problem (37), under condition (38), is proved in [6], [19]. The $L^{\infty}$-bounds for the solution were established in [8].

We also assume a periodic distribution of charges $\Sigma^{*} \equiv \Sigma^{*}(x / \varepsilon)$. Then, by periodicity of $\Omega^{\varepsilon}$ and by uniqueness of the solution $\Psi^{0, \varepsilon}$ of the Poisson-Boltzmann equation (37), we have

$$
\Psi^{0, \varepsilon}(x)=\Psi^{0}\left(\frac{x}{\varepsilon}\right), \quad n_{j}^{0, \varepsilon}(x)=n_{j}^{0}\left(\frac{x}{\varepsilon}\right),
$$

where

$$
n_{j}^{0}(y)=n_{j}^{0}(\infty) \exp \left\{-z_{j} \Psi^{0}(y)\right\}
$$

and $\Psi^{0}(y)$ is the periodic solution for the cell Poisson-Boltzmann equation

$$
\left\{\begin{array}{l}
-\Delta \Psi^{0}=\beta \sum_{j=1}^{N} z_{j} n_{j}^{0}(\infty) e^{-z_{j} \Psi^{0}} \text { in } Y_{F} \\
\nabla \Psi^{0} \cdot \nu=-N_{\sigma} \Sigma^{*} \text { on } S \\
\Psi^{0} \text { is } Y \text { - periodic. }
\end{array}\right.
$$

neutroel Remark 4. The bulk electroneutrality condition (38) is not a restriction. Actually all our results hold under the weaker assumption (7) that all valencies $z_{j}$ do not have the same sign. As explained in [6] a simple change of reference values for the potential $\psi$ and the infinite dilution constants $n_{j}^{0}(\infty)$ allows us to recover (38) for these new variables. 
At equilibrium, the pressure in Stokes equations (26) (corresponding to a zero velocity) is given (up to an additive constant) by

$$
p^{0, \varepsilon}=\sum_{j=1}^{N} n_{j}^{0}(\infty) \exp \left\{-z_{j} \Psi^{0, \varepsilon}\right\},
$$

thanks to (14) and the fact that $\frac{\partial \mathbf{u}^{0, \varepsilon}}{\partial t}=0$.

It remains to calculate the equilibrium displacement. As already said, we restrict ourselves to the case $\mathbf{F}_{\varepsilon} \approx I$. The equilibrium displacement problem has a non-trivial solution because of the Maxwell stress and the osmotic Donnan pressure $p^{0, \varepsilon}$. It was first studied by Moyne and Murad [24]. Following their lead, we look for a displacement of the type

$$
\mathbf{u}^{0, \varepsilon}(x)=\varepsilon \mathbf{u}_{\pi}^{1}\left(\frac{x}{\varepsilon}\right), \quad x \in \Omega_{s}^{\varepsilon},
$$

where $\mathbf{u}_{\pi}^{1}(y)$ is a $Y$-periodic function defined as the solution of

$$
\begin{gathered}
\operatorname{div}_{y}\left(A\left(e_{y}\left(\mathbf{u}_{\pi}^{1}\right)\right)=0 \quad \text { in } \quad Y_{s}, \mathbf{u}_{\pi}^{1} \text { is } Y\right. \text {-periodic, } \\
A e_{y}\left(\mathbf{u}_{\pi}^{1}\right) \nu=\left(-\sum_{j=1}^{N} n_{j}^{0}(\infty) \exp \left\{-z_{j} \Psi^{0}\right\} I+\frac{1}{\beta}\left(\nabla \Psi^{0} \otimes \nabla \Psi^{0}-\frac{1}{2}\left|\nabla \Psi^{0}\right|^{2} I\right)\right) \nu \\
\text { on } S=\partial Y_{s} \backslash \partial Y .
\end{gathered}
$$

It is easy to check that $\mathbf{u}^{0, \varepsilon}$, defined by (43), satisfies the equations (28), (29) and the interface condition (30).

Lemma 5. Problem (44)-(45) admits a solution $\mathbf{u}_{\pi}^{1} \in H^{1}\left(Y_{s}\right)^{d}$, unique up to the addition of a constant vector.

Proof. It is a classical result [24]. Existence is deduced from the Fredholm alternative since the non-homogeneous Neumann boundary data has zero average on $S=\partial Y_{s} \backslash \partial Y$. To check this last point, use Stokes divergence theorem and (14).

\section{Linearization}

We now proceed to the linearization of the electrokinetic equations (25)-(34) around the equilibrium solution computed in Section 4. We therefore assume that the external forces, namely the static electric potential $\Psi^{\text {ext }}(x)$ and the force $\mathbf{f}(x)$, are small. However, the surface charge density $\Sigma^{*}$ on the pore walls is not assumed to be small since it is part of the equilibrium problem studied in Section 4. Such a linearization process is classical in the ideal case and for a rigid porous medium (see the seminal paper [30] by O'Brien et al.) but it is new and slightly more complicated for the filtration through a deformable medium. For small exterior forces, we write the perturbed electrokinetic unknowns as

$$
\begin{gathered}
n_{i}^{\varepsilon}(x)=n_{i}^{0, \varepsilon}(x)+\delta n_{i}^{\varepsilon}(x), \quad \Psi^{\varepsilon}(x)=\Psi^{0, \varepsilon}(x)+\delta \Psi^{\varepsilon}(x), \\
\mathbf{w}^{\varepsilon}(x)=\delta \mathbf{w}^{\varepsilon}(x), \quad \mathbf{u}^{\varepsilon}(x)=\mathbf{u}^{0, \varepsilon}(x)+\delta \mathbf{u}^{\varepsilon}(x), \quad p^{\varepsilon}(x)=p^{0, \varepsilon}(x)+\delta p^{\varepsilon}(x),
\end{gathered}
$$


where $n_{i}^{0, \varepsilon}, \Psi^{0, \varepsilon}, \mathbf{u}^{0, \varepsilon}, p^{0, \varepsilon}$ are the equilibrium quantities, corresponding to $\mathbf{f}=0$ and $\Psi^{\text {ext }}=0$. The $\delta$ prefix indicates a perturbation. Since the equilibrium relative velocity vanishes $\mathbf{w}^{0, \varepsilon}=0$, we identify in the sequel $\mathbf{w}^{\varepsilon}=\delta \mathbf{w}^{\varepsilon}$.

Motivated by the form of the Boltzmann equilibrium distribution and the calculation of $n_{i}^{0, \varepsilon}$, we follow the lead of [30] and introduce the so-called ionic potential $\Phi_{i}^{\varepsilon}$ which is defined in terms of $n_{i}^{\varepsilon}$ by

$$
n_{i}^{\varepsilon}=n_{i}^{0}(\infty) \exp \left\{-z_{i}\left(\Psi^{\varepsilon}+\Phi_{i}^{\varepsilon}+\Psi^{e x t, *}\right)\right\} .
$$

At equilibrium the ionic potential vanishes $\Phi_{i}^{0, \varepsilon}=0$, thus we identify it with its perturbation, $\Phi_{i}^{\varepsilon}=\delta \Phi_{i}^{\varepsilon}$. After linearization (47) leads to

$$
\delta n_{i}^{\varepsilon}(x)=-z_{i} n_{i}^{0, \varepsilon}(x)\left(\delta \Psi^{\varepsilon}(x)+\Phi_{i}^{\varepsilon}(x)+\Psi^{e x t, *}(x)\right) .
$$

Introducing (48) into (25)-(34) and linearizing yields the following equations

$$
\begin{gathered}
-\varepsilon^{2} \Delta \delta \Psi^{\varepsilon}+\beta\left(\sum_{j=1}^{N} z_{j}^{2} n_{j}^{0, \varepsilon}(x)\right) \delta \Psi^{\varepsilon}=-\beta \sum_{j=1}^{N} z_{j}^{2} n_{j}^{0, \varepsilon}(x)\left(\Phi_{j}^{\varepsilon}+\Psi^{e x t, *}\right) \quad \text { in } \Omega^{\varepsilon}, \\
\nabla \delta \Psi^{\varepsilon} \cdot \nu=0 \text { on } \partial \Omega^{\varepsilon} \backslash \partial \Omega \\
\varepsilon^{2} \Delta \mathbf{w}^{\varepsilon}-\nabla P^{\varepsilon}=-\mathbf{f}^{*}-\sum_{j=1}^{N} z_{j} n_{j}^{0, \varepsilon}(x)\left(\nabla \Phi_{j}^{\varepsilon}+\mathbf{E}^{*}\right)-\frac{\mathcal{T}_{T}}{\mathcal{T}_{c}} \varepsilon^{2} \Delta \frac{\partial \delta \mathbf{u}^{\varepsilon}}{\partial t} \quad \text { in } \Omega^{\varepsilon}, \\
\operatorname{div}\left(\mathbf{w}^{\varepsilon}+\frac{\mathcal{T}_{T}}{\mathcal{T}_{c}} \frac{\partial \delta \mathbf{u}^{\varepsilon}}{\partial t}\right)=0 \text { in } \Omega^{\varepsilon}, \quad \mathbf{w}^{\varepsilon}=0 \text { on } \partial \Omega^{\varepsilon} \backslash \partial \Omega, \\
\frac{\mathcal{T}_{d}}{\mathcal{T}_{c}} n_{j}^{0, \varepsilon}(x) \frac{\partial \Phi_{j}^{\varepsilon}}{\partial t}-\operatorname{div}\left(n_{j}^{0, \varepsilon}(x)\left(\nabla \Phi_{j}^{\varepsilon}+\mathbf{E}^{*}+\frac{\operatorname{Pe}_{j}}{z_{j}} \mathbf{w}^{\varepsilon}\right)\right)=-\frac{\mathcal{T}_{d}}{\mathcal{T}_{c}} n_{j}^{0, \varepsilon}(x) \frac{\partial \delta \Psi^{\varepsilon}}{\partial t} \text { in } \Omega^{\varepsilon}, \\
\left(\nabla \Phi_{j}^{\varepsilon}+\mathbf{E}^{*}\right) \cdot \nu=0 \text { on } \partial \Omega^{\varepsilon} \backslash \partial \Omega, \\
\mathbf{w}^{\varepsilon}, P^{\varepsilon}, \Phi_{j}^{\varepsilon} \text { are } L-\text { periodic, }
\end{gathered}
$$

where, for convenience, we introduced a global pressure $P^{\varepsilon}$

$$
P^{\varepsilon}=\delta p^{\varepsilon}+\sum_{j=1}^{N} z_{j} n_{j}^{0, \varepsilon}\left(\delta \Psi^{\varepsilon}+\Phi_{j}^{\varepsilon}+\Psi^{e x t, *}\right) .
$$

It is important to remark that now, even after the global pressure $P^{\varepsilon}$ has been introduced, $\delta \Psi^{\varepsilon}$ enters equations (51)-(55) and thus is coupled to the main unknowns $\delta \mathbf{u}^{\varepsilon}, P^{\varepsilon}$ and $\Phi_{i}^{\varepsilon}$ (contrary to the case of a rigid porous medium). It remains to write the linearized equations for the displacements:

$$
\begin{gathered}
-\operatorname{div}\left(A e\left(\delta \mathbf{u}^{\varepsilon}\right)\right)=\mathbf{f}^{*} \quad \text { in } \quad \Omega_{s}^{\varepsilon}, \\
A e\left(\delta \mathbf{u}^{\varepsilon}\right) \cdot \nu=\left(-\delta p^{\varepsilon} I+2 \varepsilon^{2} e\left(\mathbf{w}^{\varepsilon}\right)+2 \frac{\mathcal{T}_{T}}{\mathcal{T}_{c}} \varepsilon^{2} e\left(\frac{\partial \delta \mathbf{u}^{\varepsilon}}{\partial t}\right)+\right. \\
\left.\frac{\varepsilon^{2}}{\beta}\left(\nabla \Psi^{0, \varepsilon} \otimes \nabla \delta \Psi^{\varepsilon}+\nabla \delta \Psi^{\varepsilon} \otimes \nabla \Psi^{0, \varepsilon}-\nabla \Psi^{0, \varepsilon} \cdot \nabla \delta \Psi^{\varepsilon} I\right)\right) \nu \quad \text { on } \quad \partial \Omega^{\varepsilon} \backslash \partial \Omega, \\
\delta \mathbf{u}^{\varepsilon} \text { is } L \text {-periodic. }
\end{gathered}
$$


Because the forcing term $\mathbf{f}^{*}$ appears in both the fluid and solid equations, while the normal stress is continuous at the fluid-solid interface, and since the boundary conditions are of periodic type, the force must satisify the following compatibility condition

$$
\int_{\Omega} \mathbf{f}^{*} d x=0
$$

\section{Choice of the time scale in the linearized model}

As explained in Remark 3 we have three time scales in the problem. The goal of this section is to discuss and compare these time scales.

Recall that, according to Table 1 , we have $\mathcal{T}_{T} / \mathcal{T}_{d}=\left(\eta D^{0}\right) /\left(\Lambda \ell^{2}\right) \approx 1 e-5$. Therefore the diffusive time scale $\mathcal{T}_{d}$ is much larger than Terzaghi's time scale $\mathcal{T}_{T}=\eta /\left(\varepsilon^{2} \Lambda\right)$, namely $\mathcal{T}_{d}>>\mathcal{T}_{T}$. Our motivation is the study of nuclear waste storage which are buried in some deep geological layers. The worst radioactive waste has a half life of about $10^{5}$ to $10^{6}$ years. Therefore we choose the characteristic time scale to be of this order, $\mathcal{T}_{c} \approx 10^{5}$ years. It is clearly much larger than $\mathcal{T}_{T}$ which is linked to the vibration period of the fluid-structure system. Our choice $\mathcal{T}_{c}>>\mathcal{T}_{T}$ simplifies a lot the analysis since the time derivative disappears in (25) and (27). Then, the structure deformation is weakly coupled to the electrokinetic model. More precisely, we first solve the electrokinetic model (independently of the elastic deformation) and in a second step we compute the elastic displacement which depends on the electrokinetic flow.

It remains to compare the diffusive time scale $\mathcal{T}_{d}$ with our choice of the characteristic time scale $\mathcal{T}_{c}$. Remember that, from definition (24), we have $\mathcal{T}_{d}=\mathcal{L}_{c}^{2} / D^{0}$ where $\mathcal{L}_{c}$ is the observation length. To define $\mathcal{L}_{c}$ we introduce a so-called observation time scale $\mathcal{T}_{\text {obs }}$ which is related to $\mathcal{L}_{c}$ through the characteristic velocity $v_{c}$ defined by $(22)$

$$
\mathcal{L}_{c}=v_{c} \mathcal{T}_{\text {obs }}=\ell \sqrt{\frac{p_{c} \mathcal{T}_{\text {obs }}}{\eta}} .
$$

In other words the observation time coincides with the convective time. At least in its early life, the waste disposal will be observed on a yearly basis. Therefore we choose the observation time to be $\mathcal{T}_{\text {obs }}=1$ year $\approx 3.15 \mathrm{e} 7 \mathrm{sec}$. Looking at the data from Table 1 , we find out $p_{c}=3 e 5$ Pa which implies that $\mathcal{L}_{c} \approx 0.15 \mathrm{~m}$. Next, by virtue of (24) we find $\mathcal{T}_{d}=0.32 \mathrm{e} 7 \mathrm{sec}$, which is roughly 1 month. Thus, we conclude that $\mathcal{T}_{c}>>\mathcal{T}_{d}$, which will be our standing assumption in the sequel. Note that this conclusion would be the same if we had chosen the observation length $\mathcal{L}_{c}$ to be of the order of the size of a single waste package (typically $1 \mathrm{~m}$ ). With the chosen scaling $\mathcal{T}_{c}>>\mathcal{T}_{d}$ we are back to the results of the articles [6] and [7]. The swelling of the elastic structure is calculated a posteriori.

Remark 6. Another possible scaling of time is $\mathcal{T}_{c} \approx \mathcal{T}_{d}$ (it could correspond, for example, to identify $\mathcal{T}_{c}$ with $\left.\mathcal{T}_{\text {obs }}\right)$. In such a case, there is still a weak coupling between the deformability of the structure and the electrokinetic system. However, this case is more complicated than the previous one $\left(\mathcal{T}_{c}>>\right.$ $\left.\mathcal{T}_{d}\right)$ because equation (49) for the potential perturbation $\delta \Psi^{\varepsilon}$ remains coupled to the ionic potential equation (53).

The above discussion shows that by neglecting all terms multiplied by the factors $\mathcal{T}_{T} / \mathcal{T}_{c}$ and $\mathcal{T}_{d} / \mathcal{T}_{c}$, we obtain a simplified system in lieu of (51)-(55). 
lem.time Lemma 7. Assume that the physical parameters are such that $\mathcal{T}_{T}<<\mathcal{T}_{c}$ and $\mathcal{T}_{d}<<\mathcal{T}_{c}$. In the limit when the ratio $\mathcal{T}_{T} / \mathcal{T}_{c}$ and $\mathcal{T}_{d} / \mathcal{T}_{c}$ vanish, system (51)-(55) simplifies to

$$
\begin{gathered}
\varepsilon^{2} \Delta \mathbf{w}^{\varepsilon}-\nabla P^{\varepsilon}=-\mathbf{f}^{*}-\sum_{j=1}^{N} z_{j} n_{j}^{0, \varepsilon}(x)\left(\nabla \Phi_{j}^{\varepsilon}+\mathbf{E}^{*}\right) \quad \text { in } \Omega^{\varepsilon}, \\
\operatorname{div} \mathbf{w}^{\varepsilon}=0 \text { in } \Omega^{\varepsilon}, \quad \mathbf{w}^{\varepsilon}=0 \quad \text { on } \partial \Omega^{\varepsilon} \backslash \partial \Omega, \\
-\operatorname{div}\left(n_{j}^{0, \varepsilon}(x)\left(\nabla \Phi_{j}^{\varepsilon}+\mathbf{E}^{*}+\frac{P e_{j}}{z_{j}} \mathbf{w}^{\varepsilon}\right)\right)=0 \text { in } \Omega^{\varepsilon}, \\
\left(\nabla \Phi_{j}^{\varepsilon}+\mathbf{E}^{*}\right) \cdot \nu=0 \text { on } \partial \Omega^{\varepsilon} \backslash \partial \Omega, \\
\mathbf{w}^{\varepsilon}, P^{\varepsilon}, \Phi_{j}^{\varepsilon} \text { are } L-\text { periodic. }
\end{gathered}
$$

The decoupled system (61)-(65) is exactly the one obtained in the case of a rigid porous medium, studied in [6]. Therefore, the deformation of the porous medium is weakly coupled to the electrokinetic flow since we first homogenize (61)-(65) and later homogenize (49)-(50) for $\delta \Psi^{\varepsilon}$ and (57)-(59) for the solid displacement $\delta \mathbf{u}^{\varepsilon}$.

\section{Homogenization of the decoupled electrokinetic system}

The homogenization of (61)-(65) is completely identical to that of the electrokinetics system for a rigid porous medium. It was already performed in our previous work [6] and we merely recall its results for the sake of completeness.

We first recall the variational formulation of (61)-(65) which relies on the following functional space for the velocity

$$
H^{\varepsilon}=\left\{\mathbf{z} \in H^{1}\left(\Omega^{\varepsilon}\right)^{d}, \operatorname{div} \mathbf{z}=0 \text { in } \Omega^{\varepsilon}, \mathbf{z}=0 \text { on } \partial \Omega^{\varepsilon} \backslash \partial \Omega, L-\text { periodic in } x\right\},
$$

and for the ionic potential

$$
H_{\#}^{1}\left(\Omega^{\varepsilon}\right)=\left\{\phi \in H^{1}\left(\Omega^{\varepsilon}\right), L-\text { periodic in } x\right\} .
$$

Introducing a bilinear form $a(\cdot, \cdot)$ and a linear form $\mathfrak{L}(\cdot)$, it reads: find $\mathbf{w}^{\varepsilon} \in H^{\varepsilon}$ and $\left\{\Phi_{j}^{\varepsilon}\right\}_{j=1, \ldots, N} \in$ $H_{\#}^{1}\left(\Omega^{\varepsilon}\right)^{N}$, such that

$$
\begin{aligned}
a\left(\left(\mathbf{w}^{\varepsilon},\left\{\Phi_{j}^{\varepsilon}\right\}\right),\left(\xi,\left\{\phi_{j}\right\}\right)\right):= & \varepsilon^{2} \int_{\Omega^{\varepsilon}} \nabla \mathbf{w}^{\varepsilon}: \nabla \xi d x+\sum_{j=1}^{N} z_{j} \int_{\Omega^{\varepsilon}} n_{j}^{0, \varepsilon}\left(\mathbf{w}^{\varepsilon} \cdot \nabla \phi_{j}-\xi \cdot \nabla \Phi_{j}^{\varepsilon}\right) d x \\
& +\sum_{j=1}^{N} \frac{z_{j}^{2}}{\mathrm{Pe}_{j}} \int_{\Omega^{\varepsilon}} n_{j}^{0, \varepsilon} \nabla \Phi_{j}^{\varepsilon} \cdot \nabla \phi_{j} d x \\
=\mathfrak{L}\left(\xi,\left\{\phi_{j}\right\}\right):= & \sum_{j=1}^{N} z_{j} \int_{\Omega^{\varepsilon}} n_{j}^{0, \varepsilon} \mathbf{E}^{*} \cdot\left(\xi-\frac{z_{j}}{\mathrm{Pe}_{j}} \nabla \phi_{j}\right) d x+\int_{\Omega^{\varepsilon}} \mathbf{f}^{*} \cdot \xi d x
\end{aligned}
$$

for any test functions $\xi \in H^{\varepsilon}$ and $\left\{\phi_{j}\right\}_{j=1, \ldots, N} \in H_{\#}^{1}\left(\Omega^{\varepsilon}\right)^{N}$.

Lemma 8. (see [6]) Let $\mathbf{E}^{*}$ and $\mathbf{f}^{*}$ be given elements of $L^{2}(\Omega)^{d}$. The variational formulation (66) admits a unique solution $\left(\mathbf{w}^{\varepsilon},\left\{\Phi_{j}^{\varepsilon}\right\}\right) \in H^{\varepsilon} \times H_{\#}^{1}\left(\Omega^{\varepsilon}\right)^{d}$, such that $\int_{\Omega^{\varepsilon}} \Phi_{j}^{\varepsilon}(x) d x=0$. Furthermore, 
there exists a constant $C$, which does not depend on $\varepsilon, \mathbf{f}^{*}$ and $\mathbf{E}^{*}$, such that the solution satisfies the following a priori estimate

$$
\left\|\mathbf{w}^{\varepsilon}\right\|_{L^{2}\left(\Omega^{\varepsilon}\right)^{d}}+\varepsilon\left\|\nabla \mathbf{w}^{\varepsilon}\right\|_{L^{2}\left(\Omega^{\varepsilon}\right)^{d^{2}}}+\max _{1 \leq j \leq N}\left\|\Phi_{j}^{\varepsilon}\right\|_{H^{1}\left(\Omega^{\varepsilon}\right)} \leq C\left(\left\|\mathbf{E}^{*}\right\|_{L^{2}(\Omega)^{d}}+\left\|\mathbf{f}^{*}\right\|_{L^{2}(\Omega)^{d}}\right) .
$$

All unknowns (velocity, pressure and ionic potentials) are merely defined in the fluid part $\Omega^{\varepsilon}$ of the porous domain. It is convenient to extend them to the whole domain $\Omega$. For simplicity we keep the same notation for the functions in $\Omega^{\varepsilon}$ and their extensions in $\Omega$. This is not difficult for the velocity $\mathbf{w}^{\varepsilon}$ which we extend by 0 in the solid part. For the ionic potential $\Phi_{j}^{\varepsilon}$ we use the extension operator introduced in [1] and which is uniformly bounded from $H^{1}\left(\Omega^{\varepsilon}\right)$ in $H^{1}(\Omega)$. For the pressure field we use the extension proposed in [38] (see also [5]).

In the present periodic setting the appropriate convergence is the two-scale convergence [4], [29]. We just recall its definition.

Def1.11. Definition 9. A sequence $\left\{w^{\varepsilon}\right\} \subset L^{2}(\Omega)$ is said to two-scale converge to a limit $w \in L^{2}(\Omega \times Y)$ if $\left\|w^{\varepsilon}\right\|_{L^{2}(\Omega)} \leq C$, and for any $\varphi \in C_{0}^{\infty}\left(\Omega ; C_{\#}^{\infty}(Y)\right)$ (\# denotes 1-periodicity) one has

$$
\lim _{\varepsilon \rightarrow 0} \int_{\Omega} w^{\varepsilon}(x) \varphi\left(x, \frac{x}{\varepsilon}\right) d x=\int_{\Omega} \int_{Y} w(x, y) \varphi(x, y) d y d x
$$

Using the a priori estimates and the notion of two-scale convergence, we were able to prove in [6] our main convergence result for the solutions of system (61)-(65).

1.15 Theorem 10. (see [6]) Let $n_{j}^{0, \varepsilon}$ be given by (39), $\left\{\mathbf{w}^{\varepsilon},\left\{\Phi_{j}^{\varepsilon}\right\}_{j=1, \ldots, N}\right\}$ be the variational solution of (66) and $P^{\varepsilon}$ be the corresponding pressure in (61). Then there exist limits $\left(\mathbf{w}^{0}, P^{0}\right) \in L^{2}\left(\Omega ; H_{\#}^{1}(Y)^{d}\right) \times$ $L^{2}(\Omega)$ and $\left\{\Phi_{j}^{0}, \Phi_{j}^{1}\right\}_{j=1, \ldots, N} \in\left(H^{1}(\Omega) \times L^{2}\left(\Omega ; H_{\#}^{1}(Y)\right)\right)^{N}$ such that the following convergences hold

$$
\begin{gathered}
\mathbf{w}^{\varepsilon} \rightarrow \mathbf{w}^{0}(x, y) \quad \text { in the two-scale sense } \\
\varepsilon \nabla \mathbf{w}^{\varepsilon} \rightarrow \nabla_{y} \mathbf{w}^{0}(x, y) \quad \text { in the two-scale sense } \\
P^{\varepsilon} \rightarrow P^{0}(x) \quad \text { strongly in } L^{2}(\Omega)
\end{gathered}
$$

Furthermore, $\left(\mathbf{w}^{0}, P^{0},\left\{\Phi_{j}^{0}, \Phi_{j}^{1}\right\}\right)$ is the unique solution of the corresponding two-scale homogenized problem.

We do not give the detailed two-scale homogenized problem (see [6] if necessary) but we rather give the following decomposition of the limits in Theorem 10, as proved in [6],

$$
\begin{aligned}
\mathbf{w}^{0}(x, y) & =\sum_{k=1}^{d}\left(-\mathbf{v}^{0, k}(y)\left(\frac{\partial P^{0}}{\partial x_{k}}-f_{k}^{*}\right)(x)+\sum_{i=1}^{N} \mathbf{v}^{i, k}(y)\left(E_{k}^{*}+\frac{\partial \Phi_{i}^{0}}{\partial x_{k}}\right)(x)\right), \\
p^{1}(x, y) & =\sum_{k=1}^{d}\left(-\pi^{0, k}(y)\left(\frac{\partial P^{0}}{\partial x_{k}}-f_{k}^{*}\right)(x)+\sum_{i=1}^{N} \pi^{i, k}(y)\left(E_{k}^{*}+\frac{\partial \Phi_{i}^{0}}{\partial x_{k}}\right)(x)\right), \\
\Phi_{j}^{1}(x, y) & =\sum_{k=1}^{d}\left(-\theta_{j}^{0, k}(y)\left(\frac{\partial P^{0}}{\partial x_{k}}-f_{k}^{*}\right)(x)+\sum_{i=1}^{N} \theta_{j}^{i, k}(y)\left(E_{k}^{*}+\frac{\partial \Phi_{i}^{0}}{\partial x_{k}}\right)(x)\right),
\end{aligned}
$$


where we introduced two family of cell problems, indexed by $k \in\{1, \ldots, d\}$. We denote by $\left\{\mathbf{e}^{k}\right\}_{1 \leq k \leq d}$ the canonical basis of $\mathbb{R}^{d}$.

The first cell problem, corresponding to the macroscopic pressure gradient, is

$$
\begin{gathered}
-\Delta_{y} \mathbf{v}^{0, k}(y)+\nabla_{y} \pi^{0, k}(y)=\mathbf{e}^{k}+\sum_{j=1}^{N} z_{j} n_{j}^{0}(y) \nabla_{y} \theta_{j}^{0, k}(y) \text { in } Y_{F} \\
\operatorname{div}_{y} \mathbf{v}^{0, k}(y)=0 \quad \text { in } Y_{F}, \quad \mathbf{v}^{0, k}(y)=0 \quad \text { on } S \\
-\operatorname{div}_{y}\left(n_{j}^{0}(y)\left(\nabla_{y} \theta_{j}^{0, k}(y)+\frac{\operatorname{Pe}_{j}}{z_{j}} \mathbf{v}^{0, k}(y)\right)\right)=0 \text { in } Y_{F} \\
\nabla_{y} \theta_{j}^{0, k}(y) \cdot \nu=0 \text { on } S .
\end{gathered}
$$

StokesAux0

$\operatorname{divAux0}$

DiffAux0

bcAux0

The second cell problem, corresponding to the macroscopic electric field, is for each species $i \in$ $\{1, \ldots, N\}$

$$
\begin{gathered}
-\Delta_{y} \mathbf{v}^{i, k}(y)+\nabla_{y} \pi^{i, k}(y)=\sum_{j=1}^{N} z_{j} n_{j}^{0}(y)\left(\delta_{i j} \mathbf{e}^{k}+\nabla_{y} \theta_{j}^{i, k}(y)\right) \text { in } Y_{F} \\
\operatorname{div}_{y} \mathbf{v}^{i, k}(y)=0 \quad \text { in } Y_{F}, \quad \mathbf{v}^{i, k}(y)=0 \quad \text { on } S, \\
-\operatorname{div}_{y}\left(n_{j}^{0}(y)\left(\delta_{i j} \mathbf{e}^{k}+\nabla_{y} \theta_{j}^{i, k}(y)+\frac{\mathrm{Pe}_{j}}{z_{j}} \mathbf{v}^{i, k}(y)\right)\right)=0 \quad \text { in } Y_{F} \\
\left(\delta_{i j} \mathbf{e}^{k}+\nabla_{y} \theta_{j}^{i, k}(y)\right) \cdot \nu=0 \text { on } S,
\end{gathered}
$$

where $\delta_{i j}$ is the Kronecker symbol. As usual the cell problems are complemented with periodic boundary conditions.

We now average (73)-(75) with respect to $y$ in order to get a purely macroscopic homogenized problem. To start with, we recall our definition of the chemical potential $\mu_{j}^{\varepsilon}=\mu_{j}^{0, \varepsilon}+\ln n_{j}^{\varepsilon}$ which is the scaled version of (3). Following Remark 1 in [6], it turns out that, for the homogenized problem, it is better to use the total electrochemical potential defined by $\mu_{j}^{\varepsilon, T}=\mu_{j}^{\varepsilon}+z_{i} \Psi^{\varepsilon}$. Its small perturbation is thus

$$
\delta \mu_{j}^{\varepsilon, T}=\delta \mu_{j}^{\varepsilon}+z_{j} \delta \Psi^{\varepsilon}=-z_{j}\left(\Phi_{j}^{\varepsilon}+\Psi^{e x t, *}\right) .
$$

Next we introduce the ionic flux of the $j$ th species

$$
\mathbf{j}_{j}^{\varepsilon}=\frac{z_{j}}{\mathrm{Pe}_{j}} n_{0, j}^{\varepsilon}\left(\nabla \Phi_{j}^{\varepsilon}+\mathbf{E}^{*}+\frac{\mathrm{Pe}_{j}}{z_{j}} \mathbf{w}^{\varepsilon}\right),
$$

where $\mathbf{E}^{*}=\nabla \Psi^{e x t, *}$. We then define their averages which will be the effective quantities

$$
\begin{gathered}
\mu_{j}^{0, T}(x)=-z_{j}\left(\Phi_{j}^{0}(x)+\Psi^{e x t, *}(x)\right), \\
\mathbf{j}_{j}^{0}(x)=\frac{z_{j}}{\mathrm{Pe}_{j}\left|Y_{F}\right|} \int_{Y_{F}} n_{j}^{0}(y)\left(\nabla_{x} \Phi_{j}^{0}(x)+\mathbf{E}^{*}+\nabla_{y} \Phi_{j}^{1}(x, y)+\frac{\mathrm{Pe}_{j}}{z_{j}} \mathbf{w}^{0}(x, y)\right) d y, \\
\mathbf{w}(x)=\frac{1}{\left|Y_{F}\right|} \int_{Y_{F}} \mathbf{w}^{0}(x, y) d y .
\end{gathered}
$$

Note that, for the ease of reading, we dropped the $\delta$ prefix. We are now able to write the homogenized or upscaled equations for the above effective fields. 
prop.eff Proposition 11. (see [6]) Define the gradient vector of pressure and total chemical potentials, $\mathcal{F}=\left(\nabla_{x} P^{0},\left\{\nabla_{x} \mu_{j}^{0, T} / z_{i}\right\}\right)$, and the gradient of mass and ionic fluxes $\mathcal{J}=\left(\mathbf{w},\left\{\mathbf{j}_{j}^{0}\right\}\right)$. The effective equations are

$$
\begin{aligned}
& \operatorname{div}_{x} \mathcal{J}=0 \quad \text { in } \Omega, \\
& \mathcal{J}=-\mathcal{M F}+\mathcal{M}\left(\mathbf{f}^{*},\{0\}\right), \\
& P^{0}, \Phi_{j}^{0} \quad L-\text { periodic, }
\end{aligned}
$$

where the overall tensor $\mathcal{M}$ is symmetric positive definite, defined by

$$
\mathcal{M}=\left(\begin{array}{cccc}
\mathbb{K} & \frac{\mathbb{J}_{1}}{z_{1}} & \cdots & \frac{\mathbb{J}_{N}}{z_{N}} \\
\mathbb{L}_{1} & \frac{\mathbb{D}_{11}}{z_{1}} & \cdots & \frac{\mathbb{D}_{1 N}}{z_{N}} \\
\vdots & \vdots & \ddots & \vdots \\
\mathbb{L}_{N} & \frac{\mathbb{D}_{N 1}}{z_{1}} & \cdots & \frac{\mathbb{D}_{N N}}{z_{N}}
\end{array}\right) .
$$

The blocks of $\mathcal{M}$ are defined by

$$
\begin{gathered}
\left\{\mathbb{J}_{i}\right\}_{l k}=\frac{1}{\left|Y_{F}\right|} \int_{Y_{F}} \mathbf{v}^{i, k}(y) \cdot \mathbf{e}^{l} d y, \quad\{\mathbb{K}\}_{l k}=\frac{1}{\left|Y_{F}\right|} \int_{Y_{F}} \mathbf{v}^{0, k}(y) \cdot \mathbf{e}^{l} d y, \\
\left\{\mathbb{D}_{j i}\right\}_{l k}=\frac{1}{\left|Y_{F}\right|} \int_{Y_{F}} n_{j}^{0}(y)\left(\mathbf{v}^{i, k}(y)+\frac{z_{j}}{\mathrm{Pe}_{j}}\left(\mathbf{e}^{k}+\nabla_{y} \theta_{j}^{i, k}(y)\right)\right) \cdot \mathbf{e}^{l} d y \\
\left\{\mathbb{L}_{j}\right\}_{l k}=\frac{1}{\left|Y_{F}\right|} \int_{Y_{F}} n_{j}^{0}(y)\left(\mathbf{v}^{0, k}(y)+\frac{z_{j}}{\mathrm{Pe}_{j}} \nabla_{y} \theta_{j}^{0, k}(y)\right) \cdot \mathbf{e}^{l} d y .
\end{gathered}
$$

The tensor $\mathbb{K}$ is called permeability tensor, $\mathbb{D}_{j i}$ are the electrodiffusion tensors. The symmetry of the tensor $\mathcal{M}$ is equivalent to the famous Onsager's reciprocal relations.

Remark 12. Besides the standard convergences of the microscopic variables to the effective ones, in [6] we also established the following strong convergences:

$$
\lim _{\varepsilon \rightarrow 0} \int_{\Omega^{\varepsilon}}\left|\mathbf{w}^{\varepsilon}(x)-\mathbf{w}^{0}\left(x, \frac{x}{\varepsilon}\right)\right|^{2} d x=0
$$

and

$$
\lim _{\varepsilon \rightarrow 0} \int_{\Omega^{\varepsilon}}\left|\nabla\left(\Phi_{j}^{\varepsilon}(x)-\Phi_{j}^{0}(x)-\varepsilon \Phi_{j}^{1}\left(x, \frac{x}{\varepsilon}\right)\right)\right|^{2} d x=0 .
$$

\section{Homogenization of the potential perturbation $\delta \Psi^{\varepsilon}$}

Once the main electokinetics system (61)-(65) has been homogenized, we now turn to the equations (49)-(50) for the potential perturbation $\delta \Psi^{\varepsilon}$. The corresponding variational formulation is: find $\delta \Psi^{\varepsilon} \in H_{\#}^{1}\left(\Omega^{\varepsilon}\right)$ such that

$$
\varepsilon^{2} \int_{\Omega^{\varepsilon}} \nabla \delta \Psi^{\varepsilon} \cdot \nabla \varphi d x+\beta \int_{\Omega^{\varepsilon}}\left(\sum_{j=1}^{N} z_{j}^{2} n_{j}^{0, \varepsilon}\right) \delta \Psi^{\varepsilon} \varphi d x=-\beta \sum_{j=1}^{N} z_{j}^{2} \int_{\Omega^{\varepsilon}} n_{j}^{0, \varepsilon}\left(\Phi_{j}^{\varepsilon}+\Psi^{e x t, *}\right) \varphi d x
$$

for any test functions $\varphi \in H_{\#}^{1}\left(\Omega^{\varepsilon}\right)$. Using the energy inequality for (88), it is straightforward to prove the following a priori estimate. 
APRIORI2 Lemma 13. Let $\Psi^{\text {ext,*}}, \mathbf{E}^{*}$ and $\mathbf{f}^{*}$ be given elements of $L^{2}(\Omega)$. The variational formulation (88) admits a unique solution $\delta \Psi^{\varepsilon} \in H_{\#}^{1}\left(\Omega^{\varepsilon}\right)$. Furthermore, there exists a constant $C$, which does not depend on $\varepsilon, \mathbf{f}^{*}$ and $\Psi^{\text {ext,*}}$, such that the solution satisfies the following a priori estimates

$$
\varepsilon^{2}\left\|\nabla \delta \Psi^{\varepsilon}\right\|_{L^{2}\left(\Omega^{\varepsilon}\right)^{d}}^{2}+\left\|\delta \Psi^{\varepsilon}\right\|_{L^{2}\left(\Omega^{\varepsilon}\right)}^{2} \leq C\left(\left\|\Psi^{e x t, *}\right\|_{H^{1}(\Omega)}^{2}+\left\|\mathbf{f}^{*}\right\|_{L^{2}(\Omega)^{d}}^{2}\right) .
$$

Using the a priori estimates and the notion of two-scale convergence, we are able to prove our main convergence result for the solutions of system (49)-(50).

1.Psi Theorem 14. Let $n_{j}^{0, \varepsilon}$ be given by (39) and $\delta \Psi^{\varepsilon}$ be the variational solution of (88). Then there exist a limit $\delta \Psi^{0} \in L^{2}\left(\Omega ; H_{\#}^{1}(Y)\right)$ such that the following convergences hold

$$
\begin{aligned}
\delta \Psi^{\varepsilon} & \rightarrow \delta \Psi^{0}(x, y) \quad \text { in the two-scale sense } \\
\varepsilon \nabla \delta \Psi^{\varepsilon} & \rightarrow \nabla_{y} \delta \Psi^{0}(x, y) \quad \text { in the two-scale sense }
\end{aligned}
$$

Furthermore, $\delta \Psi^{0}(x, y)$ is the unique solution of the two-scale homogenized problem

$$
\begin{gathered}
-\Delta_{y} \delta \Psi^{0}+\beta\left(\sum_{j=1}^{N} z_{j}^{2} n_{j}^{0}(y)\right) \delta \Psi^{0}=-\beta \sum_{j=1}^{N} z_{j}^{2} n_{j}^{0}(y)\left(\Phi_{j}^{0}(x)+\Psi^{e x t, *}(x)\right) \text { in } \Omega \times Y_{F}, \\
\nabla_{y} \delta \Psi^{0}(x, y) \cdot \nu(y)=0 \text { on } \Omega \times S, \\
\delta \Psi^{0}(x, y) \text { being 1-periodic in } y \text { and } L \text {-periodic in } x .
\end{gathered}
$$

Again, it is important to separate the fast and the slow scale. To this end, we introduce the cell problems

$$
\begin{gathered}
-\Delta_{y} \xi^{j}(y)+\beta\left(\sum_{k=1}^{N} z_{k}^{2} n_{k}^{0}(y)\right) \xi^{j}(y)=-\beta z_{j}^{2} n_{j}^{0}(y) \text { in } Y_{F}, \\
\nabla_{y} \xi^{j}(y) \cdot \nu(y)=0 \text { on } \Omega \times S, \quad \xi^{j} \text { being 1-periodic in } y .
\end{gathered}
$$

Existence and uniqueness of solutions for (95)-(96) is standard. By summing these problems over $j$, we easily deduce that $\sum_{j=1}^{N} \xi^{j}(y)=-1$ in $Y_{F}$, and by the maximum principle we deduce $-1 \leq \xi^{j}(y) \leq$ 0 , for $j=1, \ldots, N$, in $Y_{F}$, while

$$
\delta \Psi^{0}(x, y)=\sum_{j=1}^{N} \xi^{j}(y)\left(\Phi_{j}^{0}(x)+\Psi^{e x t, *}(x)\right) .
$$

Finally, we obtain the strong convergence of this sequence.

Proposition 15. The following strong two-scale convergences hold

$$
\lim _{\varepsilon \rightarrow 0} \int_{\Omega^{\varepsilon}} \varepsilon^{2}\left|\nabla\left(\delta \Psi^{\varepsilon}(x)-\delta \Psi^{0}\left(x, \frac{x}{\varepsilon}\right)\right)\right|^{2} d x=0
$$

and

$$
\lim _{\varepsilon \rightarrow 0} \int_{\Omega^{\varepsilon}}\left|\delta \Psi^{\varepsilon}(x)-\delta \Psi^{0}\left(x, \frac{x}{\varepsilon}\right)\right|^{2} d x=0 .
$$

STvelocityv 
Proof. This is an easy consequence of the following energy convergence

$$
\begin{gathered}
\lim _{\varepsilon \rightarrow 0} \varepsilon^{2} \int_{\Omega^{\varepsilon}}\left|\nabla \delta \Psi^{\varepsilon}\right|^{2} d x=\int_{\Omega} \int_{Y_{F}}\left|\nabla_{y} \delta \Psi^{0}(x, y)\right|^{2} d x d y \\
\lim _{\varepsilon \rightarrow 0} \int_{\Omega^{\varepsilon}}\left(\sum_{k=1}^{N} z_{k}^{2} n_{k}^{0, \varepsilon}\right)\left|\delta \Psi^{\varepsilon}\right|^{2} d x=\int_{\Omega} \int_{Y_{F}}\left(\sum_{k=1}^{N} z_{k}^{2} n_{k}^{0}(y)\right)\left|\delta \Psi^{0}(x)\right|^{2} d x d y
\end{gathered}
$$

and of the notion of strong two-scale convergence [4]. The energy convergences are obtained by simply passing to the limit in (88) with the test function $\delta \Psi^{\varepsilon}$ and using the weak lower semi-continuity of two-scale convergence.

\section{Homogenization of the displacement perturbation $\delta \mathbf{u}^{\varepsilon}$}

It remains to homogenize the linearized equation for the displacement. To simplify the notations in this section, we denote by $\mathbf{u}^{\varepsilon}$ the perturbation $\delta \mathbf{u}^{\varepsilon}$. We recall equations (57)-(59):

$$
\begin{gathered}
-\operatorname{div}\left(A e\left(\mathbf{u}^{\varepsilon}\right)\right)=\mathbf{f}^{*} \quad \text { in } \quad \Omega_{s}^{\varepsilon}, \\
A e\left(\mathbf{u}^{\varepsilon}\right) \cdot \nu=\sigma^{f 1, \varepsilon} \nu \quad \text { on } \quad \partial \Omega^{\varepsilon} \backslash \partial \Omega,
\end{gathered}
$$

$$
\mathbf{u}^{\varepsilon} \text { is } L \text {-periodic. }
$$

In the boundary condition (103) we used the fluid stress tensor defined by

$$
\begin{gathered}
\sigma^{f 1, \varepsilon}=-P^{\varepsilon} I+2 \varepsilon^{2} e\left(\mathbf{w}^{\varepsilon}\right)+\sum_{j=1}^{N} z_{j} n_{j}^{0, \varepsilon}\left(\delta \Psi^{\varepsilon}+\Phi_{j}^{\varepsilon}+\Psi^{e x t, *}\right) I \\
+\frac{\varepsilon^{2}}{\beta}\left(\nabla \Psi^{0, \varepsilon} \otimes \nabla \delta \Psi^{\varepsilon}+\nabla \delta \Psi^{\varepsilon} \otimes \nabla \Psi^{0, \varepsilon}-\nabla \Psi^{0, \varepsilon} \cdot \nabla \delta \Psi^{\varepsilon} I\right),
\end{gathered}
$$

which is the linearization of the fluid stress tensor $\sigma^{f, \varepsilon}$, defined by (25). The corresponding variational form is: find $\mathbf{u}^{\varepsilon} \in H_{\#}^{1}\left(\Omega_{s}^{\varepsilon}\right)^{d}$, such that

$$
\int_{\Omega_{s}^{\varepsilon}} A e\left(\mathbf{u}^{\varepsilon}\right): e(\varphi) d x=\int_{\partial \Omega^{\varepsilon} \backslash \partial \Omega} \sigma^{f 1, \varepsilon} \nu \cdot \varphi d S+\int_{\Omega_{s}^{\varepsilon}} \mathbf{f}^{*} \cdot \varphi d x
$$

for any test functions $\varphi \in H_{\#}^{1}\left(\Omega_{s}^{\varepsilon}\right)^{d}$.

As in the scalar case, it is comfortable to work with functions defined on the entire domain $\Omega$. The same $H^{1}$-extension works for vector valued functions in periodic perforated domains (see [31], Sec. 4, Chapter 1). According to Theorem 4.2 in [31], there exists an extension operator $\Pi_{\varepsilon}: H^{1}\left(\Omega_{s}^{\varepsilon}\right)^{d} \rightarrow H^{1}(\Omega)^{d}$ such that, for every $\mathbf{v} \in H^{1}\left(\Omega_{s}^{\varepsilon}\right)^{d}$, we have

$$
\begin{gathered}
\Pi_{\varepsilon} \mathbf{z}=\mathbf{z}, \quad \forall \text { rigid motions } \mathbf{z}, \quad\left\|\Pi_{\varepsilon} \mathbf{v}\right\|_{H^{1}(\Omega)^{d}} \leq C_{1}\|\mathbf{v}\|_{H^{1}\left(\Omega_{s}^{\varepsilon}\right)^{d}}, \\
\left\|\Pi_{\varepsilon} \mathbf{v}\right\|_{L^{2}(\Omega)^{d}}+\left\|e\left(\Pi_{\varepsilon} \mathbf{v}\right)\right\|_{L^{2}(\Omega)^{d^{2}}} \leq C_{1}\left(\|\mathbf{v}\|_{H^{1}\left(\Omega_{s}^{\varepsilon}\right)^{d}}+\|e(\mathbf{v})\|_{L^{2}\left(\Omega_{s}^{\varepsilon}\right)^{2}}\right), \\
\left\|\nabla \Pi_{\varepsilon} \mathbf{v}\right\|_{L^{2}(\Omega)^{d}} \leq C_{1}\|\nabla \mathbf{v}\|_{H^{1}\left(\Omega_{s}^{\varepsilon}\right)^{d}}, \quad\left\|e\left(\Pi_{\varepsilon} \mathbf{v}\right)\right\|_{L^{2}(\Omega)^{d^{2}}} \leq C_{1}\|e(\mathbf{v})\|_{L^{2}\left(\Omega_{s}^{\varepsilon}\right)^{d^{2}}} .
\end{gathered}
$$

In the sequel we identify $\mathbf{u}^{\varepsilon}$ and its extension $\Pi_{\varepsilon}\left(\mathbf{u}^{\varepsilon}\right)$ and we omit writing the extension operator. 
APRIORI3 Lemma 16. Let $\Psi^{\text {ext,*}}, \mathbf{E}^{*}$ and $\mathbf{f}^{*}$ be given elements of $L^{2}(\Omega)$. The variational formulation (106) admits a unique solution $\mathbf{u}^{\varepsilon} \in H_{\#}^{1}\left(\Omega_{s}^{\varepsilon}\right)^{d}$, such that $\int_{\Omega} \mathbf{u}^{\varepsilon} d x=0$. Furthermore, there exists a constant $C$, which does not depend on $\varepsilon, \mathbf{f}^{*}$ and $\Psi^{\text {ext,*}}$, such that the solution satisfies the following a priori estimates

$$
\left\|\nabla \mathbf{u}^{\varepsilon}\right\|_{L^{2}(\Omega)^{d^{2}}}^{2}+\left\|\mathbf{u}^{\varepsilon}\right\|_{L^{2}(\Omega)^{d}}^{2} \leq C\left(\left\|\Psi^{e x t, *}\right\|_{H^{1}(\Omega)}^{2}+\left\|\mathbf{f}^{*}\right\|_{L^{2}(\Omega)^{d}}^{2}\right) .
$$

Proof. By using Stokes divergence theorem we rewrite

$$
\int_{\partial \Omega^{\varepsilon} \backslash \partial \Omega} \sigma^{f 1, \varepsilon} \nu \cdot \varphi d S=-\int_{\Omega^{\varepsilon}} \operatorname{div}\left(\sigma^{f 1, \varepsilon} \varphi\right) d x=\int_{\Omega^{\varepsilon}} \mathbf{f}^{*} \cdot \varphi d x-\int_{\Omega^{\varepsilon}} \sigma^{f 1, \varepsilon}: \nabla \varphi d x
$$

where we used equation (61) in the fluid domain. Hence, using the compatibility condition (60), we find out that the Fredholm alternative is satisfied and problem (106) admits at least one solution $\mathbf{u}^{\varepsilon} \in H_{\#}^{1}\left(\Omega_{s}^{\varepsilon}\right)^{d}$. Next we choose $\varphi=\mathbf{u}^{\varepsilon}$ as a test function and obtain the uniform bound for $e\left(\mathbf{u}^{\varepsilon}\right)$ in $L^{2}\left(\Omega_{s}^{\varepsilon}\right)^{d^{2}}$. By the property of the extension, $e\left(\mathbf{u}^{\varepsilon}\right)$ is also uniformly bounded in $L^{2}(\Omega)^{d^{2}}$. Now if we choose $\mathbf{u}^{\varepsilon}$ such that $\int_{\Omega} \mathbf{u}^{\varepsilon} d x=0$, it is a unique solution for problem (106). By Korn's inequality it satisfies inequality (107).

Using the a priori estimates and the notion of two-scale convergence, we are able to prove our main convergence result for the solutions of system (102)-(104).

1.D Theorem 17. Let $n_{j}^{0, \varepsilon}$ be given by (39) and $\mathbf{u}^{\varepsilon}$ be the variational solution of (106). Then there exist limits $\mathbf{u}^{0} \in H_{\#}^{1}(\Omega)^{d}$ and $\mathbf{u}^{1} \in L^{2}\left(\Omega ; H_{\#}^{1}(Y)^{d}\right)$ such that the following convergences hold

$$
\begin{gathered}
\mathbf{u}^{\varepsilon} \rightarrow \mathbf{u}^{0}(x) \quad \text { in the two-scale sense } \\
\nabla \mathbf{u}^{\varepsilon} \rightarrow \nabla_{x} \mathbf{u}^{0}(x)+\nabla_{y} \mathbf{u}^{1}(x, y) \quad \text { in the two-scale sense } \\
\sigma^{f 1, \varepsilon} \rightarrow \sigma^{f 1}=-P^{0}(x) I+\sum_{j=1}^{N} z_{j} n_{j}^{0}(y)\left(\delta \Psi^{0}(x, y)+\Phi_{j}^{0}(x)+\Psi^{e x t, *}(x)\right) I \\
+\frac{1}{\beta}\left(\nabla_{y} \Psi^{0}(y) \otimes \nabla_{y} \delta \Psi^{0}(x, y)+\nabla_{y} \delta \Psi^{0}(y) \otimes \nabla_{y} \Psi^{0}(y)-\nabla_{y} \Psi^{0}(y) \cdot \nabla_{y} \delta \Psi^{0}(x, y) I\right) \\
\text { in the two-scale sense }
\end{gathered}
$$

Furthermore, $\left(\mathbf{u}^{0}, \mathbf{u}^{1}\right)$ is the unique solution of the two-scale homogenized problem

$$
\begin{gathered}
-\operatorname{div}_{y}\left(A\left(e_{y}\left(\mathbf{u}^{1}\right)+e_{x}\left(\mathbf{u}^{0}\right)\right)=0 \quad \text { in } \Omega \times Y_{s},\right. \\
A\left(e_{y}\left(\mathbf{u}^{1}\right)+e_{x}\left(\mathbf{u}^{0}\right)\right) \cdot \nu(y)=\sigma^{f 1} \cdot \nu \quad \text { on } \Omega \times S, \\
\mathbf{u}^{0} \quad \text { being L-periodic in } x, \quad \int_{\Omega} \mathbf{u}^{0} d x=0, \\
\mathbf{u}^{1} \text { being 1-periodic in } y, \quad \int_{Y_{s}} \mathbf{u}^{1} d y=0, \\
-\operatorname{div}_{x} \int_{Y_{s}} A\left(e_{y}\left(\mathbf{u}^{1}\right)+e_{x}\left(\mathbf{u}^{0}\right)\right) d y=\mathbf{f}^{*}-\operatorname{div}_{x} \int_{Y_{F}} \sigma^{f 1}(x, y) d y \quad \text { in } \Omega .
\end{gathered}
$$


Again, it is important to separate the fast and the slow scale. We introduce the following cell problems. For $i, j=1, \ldots, d$, find vector valued function $\mathbf{w}^{i j} \in H_{\#}^{1}\left(Y_{s}\right)^{d}$, with $\int_{Y_{s}} \mathbf{w}^{i j}(y) d y=0$, satisfying

$$
\begin{cases}-\operatorname{div}_{y}\left\{A\left(\frac{\mathbf{e}^{i} \otimes \mathbf{e}^{j}+\mathbf{e}^{j} \otimes \mathbf{e}^{i}}{2}+e_{y}\left(\mathbf{w}^{i j}\right)\right)\right\}=0 & \text { in } Y_{s}, \\ A\left(\frac{\mathbf{e}^{i} \otimes \mathbf{e}^{j}+\mathbf{e}^{j} \otimes \mathbf{e}^{i}}{2}+e_{y}\left(\mathbf{w}^{i j}\right)\right) \nu=0 & \text { on } \partial Y_{s} \backslash \partial Y,\end{cases}
$$

and find vector valued function $\mathbf{b}^{0} \in H_{\#}^{1}\left(Y_{s}\right)^{d}, \int_{Y_{s}} \mathbf{b}^{0}(y) d y=0$, satisfying

$$
\begin{cases}-\operatorname{div}_{y}\left(A e_{y}\left(\mathbf{b}^{0}\right)\right)=0 & \text { in } Y_{s}, \\ A e_{y}\left(\mathbf{b}^{0}\right) \nu=-\nu & \text { on } \partial Y_{s} \backslash \partial Y .\end{cases}
$$

We need a third type of cell problems, linked to the ionic potentials. For $j=1, \ldots, N$, find $\kappa^{j} \in H_{\#}^{1}\left(Y_{s}\right)^{d}$, with $\int_{Y_{s}} \kappa^{j}(y) d y=0$, such that

$$
\left\{\begin{array}{l}
-\operatorname{div}_{y}\left(A\left(e_{y}\left(\kappa^{j}\right)\right)=0 \quad \text { in } Y_{s},\right. \\
A e_{y}\left(\kappa^{j}\right) \nu=\left(n_{j}^{0} I+\frac{1}{\beta}\left(\nabla_{y} \Psi^{0} \otimes \nabla_{y} \xi^{j}+\nabla_{y} \xi^{j} \otimes \nabla_{y} \Psi^{0}-\nabla_{y} \Psi^{0} \cdot \nabla_{y} \xi^{j} I\right)\right) \nu \text { on } \partial Y_{s} \backslash \partial Y .
\end{array}\right.
$$

Due to the periodicity, the problems (116), (117) and (118) have a unique solution with regularity depending only on the smoothness of the geometry. With the assumptions made, $\mathbf{w}^{i j}, \mathbf{b}^{0}$ and $\kappa^{j}$ belong to $H^{2}\left(Y_{s}\right)^{d}$.

Then we have the following scale separation formula

$$
\mathbf{u}^{1}(x, y)=\sum_{i, j=1}^{d} \mathbf{w}^{i j}(y) e_{i j}\left(\mathbf{u}^{0}(x)\right)+P^{0}(x) \mathbf{b}^{0}(y)+\sum_{j=1}^{N} \kappa^{j}(y) z_{j}\left(\Phi_{j}^{0}(x)+\Psi^{e x t, *}(x)\right)
$$

where $e_{i j}\left(\mathbf{u}^{0}\right)$ is the $(i, j)$-entry of the strain tensor $e_{x}\left(\mathbf{u}^{0}\right)$. The effective coefficients are given by

$$
\begin{gathered}
\mathcal{A}_{k l i j}^{H}=\left(\int_{Y_{s}} A\left(\frac{\mathbf{e}^{i} \otimes \mathbf{e}^{j}+\mathbf{e}^{j} \otimes \mathbf{e}^{i}}{2}+e_{y}\left(\mathbf{w}^{i j}\right)\right) d y\right)_{k l}, \\
\mathcal{B}^{H}=\int_{Y_{s}} A e_{y}\left(\mathbf{b}^{0}\right) d y,
\end{gathered}
$$

and, for $j=1, \ldots, N$,

$$
\begin{aligned}
& \mathcal{C}^{H, j}=\int_{Y_{s}} A e_{y}\left(\kappa^{j}\right) d y+\sum_{k=1}^{N} z_{k} I \int_{Y_{F}} n_{k}^{0}(y)\left(\xi^{j}(y)+\delta_{k j}\right) d y \\
& +\frac{1}{\beta} \int_{Y_{F}}\left(\nabla_{y} \psi^{0} \otimes \nabla_{y} \xi^{j}+\nabla_{y} \xi^{j} \otimes \nabla_{y} \psi^{0}-\nabla_{y} \psi^{0} \cdot \nabla_{y} \xi^{j} I\right) d y .
\end{aligned}
$$

convenergD Proposition 18. The effective equation satisfied by $\mathbf{u}^{0}$ is

$$
\begin{gathered}
-\operatorname{div}_{x}\left(\mathcal{A}^{H} e_{x}\left(\mathbf{u}^{0}\right)\right)+\operatorname{div}_{x}\left(\left(\left|Y_{F}\right| I-\mathcal{B}^{H}\right) P^{0}-\sum_{j=1}^{N} \mathcal{C}^{H, j}\left(\Phi_{j}^{0}+\Psi^{e x t, *}\right)\right)=\mathbf{f}^{*}(x) \quad \text { in } \Omega, \\
\mathbf{u}^{0} \quad \text { being L-periodic in } x, \quad \int_{\Omega} \mathbf{u}^{0} d x=0 .
\end{gathered}
$$


The fourth-order tensor $\mathcal{A}^{H}$ and the second-order tensor $\mathcal{B}^{H}$, defined by (120) and (121), respectively, are positive definite and symmetric. Furthermore, we have the following strong convergence:

$$
\lim _{\varepsilon \rightarrow 0} \int_{\Omega_{s}^{\varepsilon}}\left|e\left(\mathbf{u}^{\varepsilon}-\mathbf{u}^{0}-\varepsilon \mathbf{u}^{1}\left(x, \frac{x}{\varepsilon}\right)\right)\right|^{2} d x=0 .
$$

Proof. Inserting the value (110) of $\sigma^{f 1}$ and (119) into equation (115), avergaing in $y$ leads to the effective equation for $\mathbf{u}^{0}$. Note that, in (123), the pressure $P^{0}$ and the ionic potentials $\Phi_{j}^{0}$ are like source terms since they have already been determined in Section 7 . The proof of the symmetry and positive definiteness of $\mathcal{A}^{H}$ and $\mathcal{B}^{H}$ is classical (see [36]).

Eventually, (124) is a consequence of the notion of strong two-scale convergence [4] and of the following energy convergence

$$
\lim _{\varepsilon \rightarrow 0} \int_{\Omega_{s}^{\varepsilon}} A e\left(\mathbf{u}^{\varepsilon}\right): e\left(\mathbf{u}^{\varepsilon}\right) d x=\int_{\Omega \times Y_{s}} A\left(e_{x}\left(\mathbf{u}^{0}\right)+e_{y}\left(\mathbf{u}^{1}\right)\right):\left(e_{x}\left(\mathbf{u}^{0}\right)+e_{y}\left(\mathbf{u}^{1}\right)\right) d y d x
$$

which is obtained by simply passing to the limit in (106) with the test function $\mathbf{u}^{\varepsilon}$ and using the weak lower semi-continuity of two-scale convergence.

\section{Assembling the global homogenized problem}

The first goal of this section is assemble the global homogenized system from the previously obtained, decoupled limit equations. Second, we explain how to recover the concentrations from the ionic potentials which have been introduced in the change of variables (47). Third, we give a dimensional version of our results, which may be more useful for the more physically or numerically inclined reader.

We start by collecting the results of the previous sections and writing down the asymptotic behavior of all unknowns in non-dimensional form. These unknowns, defined in Section 3, are the electrostatic potential $\Psi^{\varepsilon}$, the species concentrations $n_{i}^{\varepsilon}$, the convective velocity $\mathbf{w}^{\varepsilon}$ and the elastic displacement $\mathbf{u}^{\varepsilon}$. Their asymptotic behavior is obtained by summing the equilibrium solutions of Section 4 to their small perturbations, solutions of the linearized equations of Section 5. Furthermore, we recall that our results are obtained under our specific choice of time scaling, explained in Section 6.

thm.main Theorem 19. Let $\Psi^{0}$ be given by (41), $n_{j}^{0}=n_{j}^{0}(\infty) \exp \left\{-z_{j} \Psi^{0}\right\}$ by (40) and $\xi^{j}$ by (95)-(96). Let $\mathbf{w}^{0}$ be the velocity given by (73), $\Phi_{j}^{0}$ be the ionic potential and $P^{0}$ the effective pressure, given by (84). Let $\mathbf{u}_{\pi}^{1}$ be the equilibrium elastic displacement, solution of (44)-(45), and $\mathbf{u}^{\mathbf{0}}$ be the perturbed elastic displacement, solution of the Biot equation (123). 
Then the obtained two-scale approximations are summarized as follows:

$$
\begin{gathered}
\Psi^{\varepsilon}(x) \approx \Psi^{0}(y)+\sum_{j=1}^{N} \xi^{j}(y)\left(\Phi_{j}^{0}(x)+\Psi^{e x t, *}(x)\right) \\
n_{i}^{\varepsilon}(x) \approx n_{i}^{0}(y)\left(1-z_{i} \sum_{j=1}^{N}\left(\xi^{j}(y)+\delta_{i j}\right)\left(\Phi_{j}^{0}(x)+\Psi^{e x t, *}(x)\right)\right) \\
\mathbf{w}^{\varepsilon}(x) \approx \mathbf{w}^{0}(x, y) \\
p^{\varepsilon}(x) \approx P^{0}(x)+\sum_{i, j=1}^{N} n_{j}^{0}(y)\left(\delta_{i j}-z_{j}\left(\xi^{i}(y)+\delta_{i j}\right)\left(\Phi_{i}^{0}(x)+\Psi^{e x t, *}(x)\right)\right) \\
\mathbf{u}^{\varepsilon}(x) \approx \varepsilon \mathbf{u}_{\pi}^{1}(y)+\mathbf{u}^{0}(x),
\end{gathered}
$$

where the variable $y$ should be replaced by $x / \varepsilon$.

Proof. The starting point is the linearization expansion (46). The leading terms (indexed with an exponent 0) in the linearization are the equilibrium solutions computed in Section 4. Concerning the perturbations (denoted with a prefix $\delta$ ), we first recall the change of variable (48) which replace $\delta n_{i}^{\varepsilon}$ by the ionic potential $\Phi_{i}^{\varepsilon}$. Then, each perturbative term has an asymptotic expansion provided by Sections 7, 8 and 9. Collecting these results yields (126)-(130).

The next step is to average, with respect to $y$, the right hand sides in Theorem 19 in order to obtain the effective or upscaled unknowns. To do so, we introduce some averaged and constant quantities

$$
\begin{gathered}
\Xi_{j}=\frac{1}{\left|Y_{F}\right|} \int_{Y_{F}} \xi^{j}(y) d y, \quad n_{j}^{m o y}=\frac{1}{\left|Y_{F}\right|} \int_{Y_{F}} n_{j}^{0}(y) d y, \quad j=1, \ldots, N, \\
\Psi^{m o y}=\frac{1}{\left|Y_{F}\right|} \int_{Y_{F}} \Psi^{0}(y) d y, \quad \mathcal{N}_{i j}=\frac{z_{i}}{\left|Y_{F}\right|} \int_{Y_{F}} n_{i}^{0}(y)\left(\xi^{j}(y)+\delta_{i j}\right) d y, \quad i, j=1, \ldots, N .
\end{gathered}
$$

We also remark that the equilibrium displacement $\mathbf{u}_{\pi}^{1}$, solution of the cell problem (44)-(45), is defined up to an additive constant. We therefore choose this constant such that

$$
\int_{Y_{s}} \mathbf{u}_{\pi}^{1}(y) d y=0 .
$$

Even if we do not make this choice, the average of the equilibrium displacement is small because it is multiplied by $\varepsilon$ in (130). From these definitions, we deduce the macroscopic quantities (in 
non-dimensionalized form)

$$
\begin{gathered}
\Psi^{\text {eff,nond }}(x)=\Psi^{\text {moy }}+\sum_{j=1}^{N} \Xi_{j}\left(\Phi_{j}^{0}(x)+\Psi^{\text {ext }, *}(x)\right), \\
n_{i}^{\text {eff,nond }}(x)=n_{i}^{\text {moy }}-\sum_{j=1}^{N} \mathcal{N}_{i j}\left(\Phi_{j}^{0}(x)+\Psi^{\text {ext }, *}(x)\right), \\
\mathbf{w}^{\text {eff,nond }}(x)=-\mathbb{K}\left(\nabla_{x} P^{0}(x)-\mathbf{f}^{*}(x)\right)+\sum_{i=1}^{N} \mathbb{J}_{i}\left(\nabla_{x} \Phi_{i}^{0}(x)+\mathbf{E}^{*}(x)\right), \\
p^{\text {eff,nond }}(x)=P^{0}(x)+\sum_{j=1}^{N} n_{j}^{\text {moy }}-\sum_{k, j=1}^{N} \mathcal{N}_{j k}\left(\Phi_{k}^{0}(x)+\Psi^{\text {ext }, *}(x)\right)=P^{0}(x)+\sum_{j=1}^{N} n_{j}^{e f f, \text { nond }}(x) \\
\mathbf{u}^{\text {eff,nond }}(x)=\mathbf{u}^{\mathbf{0}}(x) .
\end{gathered}
$$

All these physical quantities (132)-(136) depend on the unknowns $\left\{P^{0}, \mathbf{u}^{0}, \Phi_{j}^{0}\right\}$ which are found by solving successively the electrokinetic homogenized system (84) for $\left\{P^{0}, \Phi_{j}^{0}\right\}$ and the homogenized Biot equation (123) for $\mathbf{u}^{0}$.

From a physical point of view, the ionic potentials $\Phi_{j}^{0}$ are not the most convenient unknowns and one would rather replace them by some effective concentrations. We now explain how to recover the concentrations from these ionic potentials, which were introduced in the change of variables (47), and why the homogenized system (84) cannot possibly be written in terms of concentrations rather than ionic potentials.

As in the proof of Theorem 19, we start from equation (48) which, in the two-scale limit, gives

$$
\delta n_{i}(x, y)=-z_{i} n_{i}^{0}(y)\left(\delta \Psi^{0}(x, y)+\Phi_{i}^{0}(x)+\Psi^{e x t, *}(x)\right) .
$$

The limit perturbed potential $\delta \Psi^{0}(x, y)$ is a solution of the homogenized equation (92), with the boundary conditions (93) and (94). By linearity of the homogenized equation one can write

$$
\delta \Psi(x, y)=\sum_{j=1}^{N}\left(\Phi_{j}^{0}(x)+\Psi^{e x t, *}(x)\right) \xi_{j}(y)
$$

where $\xi_{j}(y)$ is a solution of equation (95) without anymore the $x$ variable. We recall the homogenized total electrochemical potential

$$
\mu_{j}^{0, T}(x)=-z_{j}\left(\Phi_{j}^{0}(x)+\Psi^{e x t, *}(x)\right) .
$$

From (137) and (138) we deduce

$$
\delta n_{i}(x, y)=n_{i}^{0}(y)\left(\mu_{i}^{0, T}(x)+\sum_{j=1}^{N} \frac{z_{i}}{z_{j}} \mu_{j}^{0, T}(x) \xi_{j}(y)\right) .
$$

Averaging (139) on the unit cell yields

$$
\delta n_{i}(x)=\frac{1}{\left|Y_{F}\right|} \int_{Y_{F}} \delta n_{i}(x, y) d y=\sum_{j=1}^{N} \frac{1}{z_{j}} \mathcal{N}_{i j} \mu_{j}^{0, T}(x),
$$


where $\mathcal{N}_{i j}$ is defined by (131). Introducing the matrix $A=\left(a_{i j}\right)$ defined by

$$
a_{i j}=\frac{\mathcal{N}_{i j}}{z_{j}}
$$

we recover the perturbed concentrations from the homogenized electrochemical potential

$$
\delta n(x)=A \mu^{0, T}(x)
$$

The key point, which prevents the use of the unknown $\delta n$ in lieu of $\mu^{0, T}$, is the following lemma.

Lemma 20. The matrix $A$ is not invertible. Furthermore, in the limit $\beta \rightarrow+\infty A$ is non-negative and its kernel is of dimension one.

Proof. If we sum the equations (95) we easily see that an obvious solution is

$$
\sum_{j=1}^{N} \xi_{j}(y)=-1
$$

Thus we deduce

$$
\sum_{j=1}^{N} \mathcal{N}_{i j}=\sum_{j=1}^{N} z_{j} a_{i j}=0
$$

In other words, the vector $\left(z_{1}, \ldots, z_{N}\right)$ belongs to the kernel of $A$.

For a vector $\lambda=\left(\lambda_{1}, \ldots, \lambda_{N}\right)$, introduce $n_{\lambda}^{0}=\sum_{j=1}^{N} \lambda_{j} z_{j}^{2} n_{j}^{0}$ and $\xi_{\lambda}=\sum_{j=1}^{N} \lambda_{j} \xi_{j}$ which is a solution of

$$
\begin{gathered}
-\Delta_{y}\left(\xi_{\lambda}\right)+\beta\left(\sum_{j=1}^{N} z_{j}^{2} n_{j}^{0}\right) \xi_{\lambda}=-\beta n_{\lambda}^{0} \text { in } Y_{F}, \\
\nabla_{y} \xi_{\lambda} \cdot \nu=0 \text { on } S, \quad y \rightarrow \xi_{\lambda}(y) \text { is } Y-\text { periodic. }
\end{gathered}
$$

One can check that the matrix $A$ is defined by

$$
A \lambda \cdot \lambda=\sum_{j=1}^{N} \lambda_{j}^{2} \int_{Y} n_{j}^{0}(y) d y+\int_{Y} n_{\tilde{\lambda}}^{0}(y) \xi_{\tilde{\lambda}}(y) d y,
$$

with $\tilde{\lambda}=\left(\lambda_{1} / z_{1}, \ldots, \lambda_{N} / z_{N}\right)$. In the limit as $\beta \rightarrow+\infty$, we now prove that the matrix $A$ is nonnegative and that its kernel is reduced to the vector $\left(z_{1}, \ldots, z_{N}\right)$. Indeed, for $\beta=+\infty$ one find

$$
\xi_{\lambda}=\frac{-n_{\lambda}^{0}}{\sum_{j=1}^{N} z_{j}^{2} n_{j}^{0}}
$$

By Cauchy-Schwartz inequality we deduce

$$
-n_{\tilde{\lambda}}^{0}(y) \xi_{\tilde{\lambda}}(y)=\frac{\left(\sum_{j=1}^{N} \tilde{\lambda}_{j} z_{j}^{2} n_{j}^{0}(y)\right)^{2}}{\sum_{j=1}^{N} z_{j}^{2} n_{j}^{0}(y)} \leq \sum_{j=1}^{N} \lambda_{j}^{2} n_{j}^{0}(y),
$$

which proves that $A \lambda \cdot \lambda \geq 0$ and equality can happen only in the case of equality in the CauchySchwartz inequality which is equivalent to the vector $\left(\lambda_{j} \sqrt{n_{j}^{0}}\right)$ being parallel to $\left(z_{j} \sqrt{n_{j}^{0}}\right)$. In other words, the kernel of $A$ is reduced to the vector $\left(z_{1}, \ldots, z_{N}\right)$. 
Remark 21. For example, consider an electrolyte with two ions $(N=2)$. Then the matrix $A$ is of the type

$$
A=a^{2}\left(\begin{array}{cc}
z_{2}^{2} & -z_{1} z_{2} \\
-z_{1} z_{2} & z_{1}^{2}
\end{array}\right) .
$$

One cannot use the perturbed concentrations $\delta n_{i}(x)$ as unknowns, in place of the total electrochemical potentials $\mu_{i}^{0, T}(x)$, since they satisfy the following electro-neutrality condition

$$
\sum_{j=1}^{N} \delta n_{j}(x)=0
$$

which is the direct consequence of the vector $\left(z_{1}, \ldots, z_{N}\right)$ belonging to the kernel of $A$.

Our last task is to rewrite the effective equations (84) (electrokinetic system) and (123) (Biot equation) in dimensional form. Following the adimensionalization choices of Section 3, we define the following dimensionalized unknowns

$$
\mathbf{u}^{\text {eff }}=u_{c}^{s} \mathbf{u}^{\text {eff,nond }}, \quad P^{e f f}=p_{c} P^{0}, \quad \Phi_{j}^{e f f}=\zeta \Phi_{j}^{0}, \quad \mathbf{w}^{\text {eff }}=v_{c} \mathbf{w}^{\text {eff,nond }} .
$$

We also introduced the dimensional Darcy permeability tensor $\mathbb{K}^{D}=\ell^{2} \mathbb{K}$ and the Gassmann elasticity tensor $\mathbb{A}^{G}=\Lambda A^{H}$. Then, we deduce

$$
\begin{gathered}
-\operatorname{div}_{x}\left\{\mathbb{A}^{G} e_{x}\left(\mathbf{u}^{e f f}\right)\right\}+\operatorname{div}_{x}\left\{\left(\left|Y_{F}\right| I-\mathcal{B}^{H}\right) P^{e f f}-e n_{c} \sum_{j=1}^{N} \mathcal{C}^{H, j}\left(\Phi_{j}^{e f f}+\Psi^{e x t}\right)\right\}=\mathbf{f} \text { in } \Omega, \\
\operatorname{div}_{x} \mathbf{w}^{e f f}=0 \quad \text { and } \quad \operatorname{div}_{x} \mathbf{j}_{j}^{e f f}=0 \quad \text { in } \Omega, \\
\mathbf{w}^{e f f}=\frac{\ell^{2} e n_{c}}{\eta} \sum_{i=1}^{N} \mathbb{I}_{i}\left(\nabla_{x} \Phi_{i}^{e f f}(x)+\mathbf{E}\right)-\frac{\mathbb{K}^{D}}{\eta}\left(\nabla_{x} P^{e f f}-\mathbf{f}\right) \quad \text { in } \Omega, \\
\mathbf{j}_{j}^{e f f}=\frac{n_{c} D_{j}^{0}}{\zeta} \sum_{i=1}^{N} \mathbb{D}_{j i}\left(\nabla_{x} \Phi_{i}^{e f f}(x)+\mathbf{E}\right)-\frac{D_{j}^{0}}{k_{B} T} \mathbb{L}_{j}\left(\nabla_{x} P^{e f f}-\mathbf{f}\right), \quad j=1, \ldots N, \quad \text { in } \Omega .
\end{gathered}
$$

We recall that Biot equation (142) for the effective displacement is decoupled from the rest of the system. Because of our scaling assumption from Section 6, our model corresponds to the long time behavior and it is static.

\section{A convenient ALE formulation}

In this section we explain how to couple the fluid and solid equations in a consistent manner. We use an ALE (Arbitrary Lagrangian Eulerian) formulation which is a classical setting in fluid-structure interaction (see e.g. [34], [40]). We first explain the coupling in full generality and then restrict our attention to small deformations of the solid skeleton of the porous medium, in order to recover the setting of Section 2.

The equations describing the deformation of the solid skeleton are usually written in Lagrangian coordinates. Let $\Omega_{s}(0)$ be the initial solid skeleton configuration and $\Omega_{s}(t)$ be its shape evolving with time $t$. We denote by $\mathbf{u}^{s}$ the solid skeleton displacement in Lagrangian coordinates, which is 
defined in $\Omega_{s}(0)$. The fluid-solid structure interface is denoted by $\Gamma(t)$. At the interface we write the velocity continuity condition

$$
\frac{\partial \mathbf{u}^{s}}{\partial t}=\mathbf{v} \text { on } \Gamma(t)
$$

where the fluid velocity is taken at a point $x \in \Gamma(t)$ and at instant $t$, but the time derivative of the solid displacement $\mathbf{u}^{s}$ should be taken at the position where that point $x$ was initially. Since the fluid part $\Omega_{p}(t)=\Omega \backslash\left(\Omega_{s}(t) \cup \Gamma(t)\right)$ varies with time, the interface condition (145), as well as the contact force continuity, are nonlocal and the formulation gets unclear.

Our remedy is to use the following framework.

(i) We assume that the solid displacement $\mathbf{u}^{s}$ is smooth and can be smoothly extended to the pore space $\Omega_{p}(0)$.

(ii) Following [34], we use Stokes equations in ALE coordinates (also called the "practicable ALE fluid problem in a fixed domain" in [40]).

(iii) We consider the coupled problem consisting of the Navier equations of linearized elasticity and a particular arbitrary Lagrangian-Eulerian formulation for the flow.

The main advantage of this framework is to have fixed interfaces.

Hence we use the Lagrangian formulation for the solid skeleton $\Omega_{s}$. Let us denote by $\xi \in \Omega_{s}(t)$ the Eulerian variable and by $x \in \Omega_{s}(0)$ the Lagrangian variable (our notations are not the usual ones because we end up with a Lagrangian formulation and for simplicity of notations we prefer to use the letter $x$ rather than $\xi$ ). The solid displacement is $\mathbf{u}^{s}=\xi-x$. As already stated, we suppose it smooth and having a smooth extension to the initial pore space $\Omega_{p}(0)$. Next we introduce the deformation gradient $\mathbf{F}=\nabla_{x} \xi$ and its Jacobian determinant as

$$
\mathbf{F}=I+\nabla_{x} \mathbf{u}^{s}, \quad J=\operatorname{det} \mathbf{F} .
$$

defgrad

After [34] the momentum equation in Lagrangian formulation reads

$$
\begin{gathered}
-\operatorname{div}_{x}\left(J \sigma^{s} \mathbf{F}^{-*}\right)=\rho_{s} \mathbf{f} \text { in } \Omega_{s}(0) \\
\sigma^{s}=J^{-1} \mathbf{F} A e\left(\mathbf{u}^{s}\right) \mathbf{F}^{*} \quad \text { with } \quad e\left(\mathbf{u}^{s}\right)=\frac{1}{2}\left(\nabla_{x} \mathbf{u}^{s}+\left(\nabla_{x} \mathbf{u}^{s}\right)^{*}\right),
\end{gathered}
$$

where $\sigma^{s}$ is the stress tensor of the solid phase and $A$ is a 4 th order symmetric positive definite tensor. As usual, we assume an isotropic tensor, $A_{i j k l}=2 \mu \delta_{i k} \delta_{j l}+\lambda \delta_{k l} \delta_{i j}$, where $\mu$ and $\lambda$ are the Lamé moduli.

Next the flow equation in the ALE formulation reads as follows:

$$
\begin{aligned}
& -\operatorname{div}_{x}\left(J \sigma^{f} \mathbf{F}^{-*}\right)=J \rho_{f} \mathbf{f} \quad \text { in } \quad \Omega_{p}(0), \\
& \operatorname{div}_{x}\left(J \mathbf{F}^{-1} \mathbf{v}\right)=0 \quad \text { in } \quad \Omega_{p}(0), \\
& \sigma^{f}=-p I+\eta\left(\nabla_{x} \mathbf{v} \mathbf{F}^{-1}+\mathbf{F}^{-*}\left(\nabla_{x} \mathbf{v}\right)^{*}\right)+\mathcal{E}\left(\mathbf{E} \otimes \mathbf{E}-\frac{1}{2}|\mathbf{E}|^{2}\right) \quad \text { in } \quad \Omega_{p}(0), \\
& \partial_{t} \mathbf{u}^{s}=\mathbf{v} \text { on } \Gamma(0), \\
& J \sigma^{f} \mathbf{F}^{-*} \nu=J \sigma^{s} \mathbf{F}^{-*} \nu \text { on } \Gamma(0 \text {. }
\end{aligned}
$$

We continue by transforming the Poisson equation for the electric potential

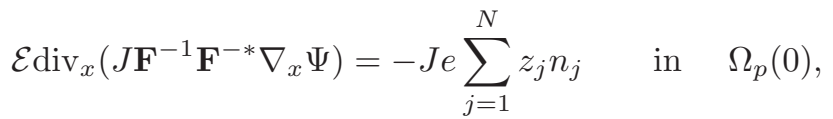


where $\mathbf{E}=-\mathbf{F}^{-1} \nabla_{x} \Psi$ is the electrical field. The boundary condition reads

$$
\mathcal{E} \mathbf{F}^{-1} \nabla_{x} \Psi \cdot \nu=-\Sigma \quad \text { on } \quad \partial \Omega_{p}(0) \backslash \partial \Omega,
$$

Finally, for the ion concentrations we have

$$
\begin{gathered}
J \frac{\partial n_{i}}{\partial t}+J \mathbf{F}^{-1}\left(\mathbf{v}-\frac{\partial \mathbf{u}^{s}}{\partial t}\right) \cdot \nabla_{x} n_{i}+\operatorname{div}_{x}\left(J \mathbf{F}^{-1} \mathbf{j}_{i}\right)=0 \quad \text { in } \quad \Omega_{p}(0), \quad i=1, \ldots, N, \\
\mathbf{j}_{i}=-\frac{D_{i}}{k_{B} T} n_{i} \mathbf{F}^{-*}\left(k_{B} T \nabla_{x} \ln n_{i}+z_{i} e \nabla_{x} \Psi\right), \quad i=1, \ldots, N . \\
J \mathbf{F}^{-1} \mathbf{j}_{i} \cdot \nu=0 \text { on } \Gamma(0) .
\end{gathered}
$$

After writing the complete pore level fluid-structure problem in this ALE Lagrangian formulation, we rely on the data of Table 1 (see Remark 2 ) to conclude that $\mathbf{F} \approx I$ and $J \approx 1$. Therefore equations (147) to (158) simplify in the equations given in Section 2.

\section{References}

ACDP [1] E. Acerbi, V. Chiadò Piat, G. Dal Maso, D. Percivale, An extension theorem from connected sets, and homogenization in general periodic domains, Nonlinear Anal., 18 (1992), 481-496.

[2] P. M. Adler, V. Mityushev, Effective medium approximation and exact formulae for electrokinetic phenomena in porous media, J. Phys. A: Math. Gen. 36 (2003), 391-404.

A:01 [3] P. M. Adler, Macroscopic electroosmotic coupling coefficient in random porous media, Math. Geol. 33(1) (2001), 63-93.

Al192 [4] G. Allaire, Homogenization and two-scale convergence, SIAM J. Math. Anal., 23 (1992), 14821518.

Al197

[5] G. Allaire, One-Phase Newtonian Flow, in Homogenization and Porous Media, ed. U.Hornung, Springer, New-York, (1997), 45-68.

[6] G. Allaire, A. Mikelić, A. Piatnitski, Homogenization of The Linearized Ionic Transport Equations in Rigid Periodic Porous Media, Journal of Mathematical Physics, 51, 123103 (2010). Erratum in the same journal, 52, 063701 (2011).

[7] G. Allaire, R. Brizzi, J.-F. Dufrêche, A. Mikelić, A. Piatnitski, Ion transport in porous media: derivation of the macroscopic equations using upscaling and properties of the effective coefficients, Comp. Geosci., 17, Issue 3, 479-495 (2013).

[8] G. Allaire, J.-F. Dufrêche, A. Mikelić, A. Piatnitski, Asymptotic analysis of the PoissonBoltzmann equation describing electrokinetics in porous media, Nonlinearity, 26 (2013) 881-910.

[9] G. Allaire, R. Brizzi, J. F. Dufrêche, A. Mikelić, A. Piatnitski: Role of non-ideality for the ion transport in porous media: derivation of the macroscopic equations using upscaling, Physica D: Nonlinear Phenomena 282 (2014), p. 39-60. doi: 10.1016/j.physd.2014.05.007.

[10] J. L. Auriault, T. Strzelecki, On the electro-osmotic flow in a saturated porous medium, Int. J. Engng Sci. 19 (1981), 915-928. 
[1.2] [11] J.-L. Auriault, Poroelastic media, in: U. Hornung (Ed.), Homogenization and Porous Media, in: Interdiscip. Appl. Math., Springer, Berlin, 1997, pp. 163-182.

ClFGM [12] Th. Clopeau, J.L. Ferrín, R.P. Gilbert, A. Mikelić : Homogenizing the Acoustic Properties of the Seabed ,II, Mathematical and Computer Modelling, Vol. 33 (2001), p. 821-841.

CSTA:96 [13] D. Coelho, M. Shapiro, J.-F. Thovert, P. M. Adler, Electro-osmotic phenomena in porous media, J. Colloid Interface Sci. 181 (1996), 169-90.

Dufreche05 [14] J.-F. Dufrêche, O. Bernard, S. Durand-Vidal, P. Turq, Analytical Theories of Transport in Concentrated Electrolyte Solutions from the MSA, J. Phys. Chem. B 109 (2005), 9873.

GCA:06 [15] A.K. Gupta, D. Coelho, P.M. Adler, Electroosmosis in porous solids for high zeta potentials, Journal of Colloid and Interface Science 303 (2006), 593-603.

OMP:07 [16] O. Iliev, A. Mikelić, P. Popov : On upscaling certain flows in deformable porous media, SIAM: Multiscale Model. Simul., Vol. 7 (2008), no. 1, p. 93-123.

JMNR11 [17] W. Jäger, A. Mikelić, M. Neuss-Radu : Homogenization-limit of a model system for interaction of flow, chemical reactions and mechanics in cell tissues, SIAM J. Math. Anal., Vol. 43, No. 3 (2011), p. 1390-1435.

$\mathrm{KBA}: 05$

[18] G. Karniadakis, A. Beskok, N. Aluru, Microflows and Nanoflows. Fundamentals and Simulation, Interdisciplinary Applied Mathematics, Vol. 29, Springer, New York (2005).

L:06 [19] J.R. Looker, Semilinear elliptic Neumann problems and rapid growth in the nonlinearity, Bull. Austral. Math. Soc., Vol. 74, no.2, (2006), 161-175.

LC:06 [20] J.R. Looker, S.L. Carnie, Homogenization of the ionic transport equations in periodic porous media, Transp. Porous Media 65 (2006), 107-131.

MSA:01 [21] S. Marino, M. Shapiro, P.M. Adler, Coupled transports in heterogeneous media, J. Colloid Interface Sci. 243 (2001), 391-419.

[A3] [22] A. Mikelić, M. F. Wheeler : On the interface law between a deformable porous medium containing a viscous fluid and an elastic body, M3AS: Mathematical Models and Methods in Applied Sciences, Vol. 22, No. 11 (2012), 1240031 (32 pages); doi: 10.1142/S0218202512500315 .

MM:02 [23] C. Moyne, M. Murad, Electro-chemo-mechanical couplings in swelling clays derived from a micro/macro-homogenization procedure, Int. J. Solids Structures 39 (2002), 6159-6190.

MM:03 [24] C. Moyne, M. Murad, Macroscopic behavior of swelling porous media derived from micromechanical analysis, Transport Porous Media 50 (2003), 127-151.

MM:06 [25] C. Moyne, M. Murad, A Two-scale model for coupled electro-chemomechanical phenomena and Onsager's reciprocity relations in expansive clays: I Homogenization analysis, Transport Porous Media 62 (2006), 333-380.

MM:06a [26] C. Moyne, M. Murad, A two-scale model for coupled electro-chemo-mechanical phenomena and Onsager's reciprocity relations in expansive clays: II. Computational validation, Transp. Porous Media 63(1) (2006), 13-56. 
MM:08 [27] C. Moyne, M. Murad, A dual-porosity model for ionic solute transport in expansive clays, Comput Geosci 12 (2008), 47-82.

[1.6] [28] J. Neev, F.R. Yeatts, Electrokinetic effects in fluid-saturated poroelastic media, Phys. Rev. B, Vol. 40 (1989), p. 9135-9141.

NGU [29] G. Nguetseng, A general convergence result for a functional related to the theory of homogenization, SIAM J. Math. Anal. 20(3), 608-623 (1989).

OBW:78 [30] R. W. O'Brien, L. R. White, Electrophoretic mobility of a spherical colloidal particle, J. Chem. Soc., Faraday Trans. 2 74(2) (1978), 1607-1626.

Olein92 [31] O. A. Oleinik, A. M. Shamaev, A. G. Yosifian, Mathematical Problems in Elasticity and Homogenization, North Holland, Amsterdam, London, New York, Tokyo, (1992).

[1.7] [32] S. Pride, Governing equations for the coupled electromagnetics and acoustics of porous media, Phys. Rev. B 50, 15678-15696 (1994).

Rayetal:11 [33] N. Ray, Ch. Eck, A. Muntean, P. Knabner, Variable Choices of Scaling in the Homogenization of a Nernst-Planck-Poisson Problem, preprint no. 344, Institut für Angewandte Mathematik, Universitaet Erlangen-Nürnberg (2011).

Richt1 [34] T. Richter, Numerical Methods for Fluid-Structure Interaction Problems, preprint, http://ganymed.iwr.uni-heidelberg.de/ richter/SS10/fsi/fsi.pdf.

RPA:06 [35] M. Rosanne, M. Paszkuta, P.M. Adler, Electrokinetic phenomena in saturated compact clays, Journal of Colloid and Interface Science, 297 (2006), 353-364.

SP80 [36] E. Sanchez-Palencia, Non-Homogeneous Media and Vibration Theory, Lecture Notes in Physics 127, Springer Verlag, (1980).

schmuck [37] M. Schmuck, Modeling And Deriving Porous Media Stokes-Poisson-Nernst-Planck Equations By A Multiple-Scale Approach, Commun. Math. Sci. 9 (2011), no. 3, 685-710.

Ta1980 [38] L. Tartar, Convergence of the Homogenization Process, Appendix of [36].

[1.3] [39] I. Tolstoy, ed., Acoustics, elasticity, and thermodynamics of porous media. Twenty-one papers by M.A. Biot, Acoustical Society of America, New York, 1992.

wick [40] T. Wick, Solving monolithic fluid-structure interaction problems in arbitrary lagrangian eulerian coordinates with the deal. II library, Archive of Numerical Software, vol. 1 (2013), no 1, p. 1-19. 